\section{Research Square}

Preprints are preliminary reports that have not undergone peer review.

They should not be considered conclusive, used to inform clinical practice, or referenced by the media as validated information.

\title{
COVID-19 Experiences Predicting High Anxiety and Depression Among a Sample of BRCA1/BRCA2-positive Women in the US
}

\author{
Kate E Dibble ( $\sim$ kdibble2@jhu.edu ) \\ Johns Hopkins Bloomberg School of Public Health \\ Avonne E Connor \\ Johns Hopkins Bloomberg School of Public Health
}

\section{Research Article}

Keywords: COVID-19, BRCA1, BRCA2, anxiety, depression, disparities

Posted Date: August 9th, 2021

DOI: https://doi.org/10.21203/rs.3.rs-763516/v1

License: () (7) This work is licensed under a Creative Commons Attribution 4.0 International License. Read Full License

Version of Record: A version of this preprint was published at Scientific Reports on December 1st, 2021. See the published version at https://doi.org/10.1038/s41598-021-04353-x. 


\section{Abstract}

Purpose. During the COVID-19 pandemic, breast and ovarian cancer survivors experienced more anxiety and depression than before the pandemic. Studies have not investigated the similarities of this trend among BRCA1/2-positive women who are considered high risk for these cancers. The current study examines the impact of COVID-19 experiences on anxiety and depression in a sample of BRCA1/2-positive women in the U.S.

Methods. 211 BRCA1/2-positive women from medically underserved backgrounds completed an online survey. Adjusted odds ratios (aORs) and 95\% confidence intervals (Cls) were estimated using multivariable logistic regression for associations between COVID-19 experiences and self-reported anxiety and depression stratified by demographic factors.

Results. Overall, women who reported quarantining/isolation (aOR, $0.46,95 \% \mathrm{Cl}, 0.24-0.88$ ) experienced significantly fewer depressive symptoms than women who did not report this experience. Racial/ethnic minority women caring for someone at home during COVID-19 were 3.78 times more likely (95\% Cl, 1.04-13.6) to report high anxiety while non-Hispanic white women were less likely $(\mathrm{aOR}, 0.36,95 \% \mathrm{Cl}, 0.10-1.33$, $p$-interaction=0.011).

Conclusions. To date, this is the first study to analyze anxiety and depression considering several COVID-19 predictors among BRCA1/2-positive women. Our findings can be used to inform future research and advise COVID-19-related mental health resources specific to these women.

\section{Introduction}

One in eight women will be diagnosed with breast cancer in their lifetime, but only $5-10 \%$ of these women have a $B R C A 1$ and/or $B R C A 2$ (BReast CAncer) genetic mutation ${ }^{1}$. Although rare, these mutations occur on dominant genes, indicative of a $50 \%$ inheritance rate, and therefore, occur within biological family units and often co-occur with other rarer cancer-specific mutations such as ATM, CDH1, CHEK2, PALB2, PTEN, STK11, and TP532. Due to these mutations, women have an increased risk of breast and ovarian cancers ${ }^{3}$, living with a cumulative breast cancer risk of $72 \%$ among $B R C A 1$ and $69 \%$ among $B R C A 2$ carriers ${ }^{4}$. Ovarian cancer risk is also elevated by the presence of these mutations, with one study finding that ovarian cancer occurs in an estimated $44 \%$ of $B R C A 1$-positive women and $17 \%$ for those with $B R C A 2^{4}$. When breast cancer does occur among this population, those with $B R C A 1$ mutations are more likely to be diagnosed with triple-negative breast cancer, associated with higher risk of mortality ${ }^{5}$, while BRCA2-positive women are more likely to be diagnosed with hormone receptor-positive tumors ${ }^{6}$. The rate of recurrence has been approximated between $25-30 \%$ remains elevated among $B R C A 1 / 2$-positive cancer survivors but remains highly dependent on individual clinical characteristics such as stage at diagnosis, treatment(s), and hormone receptor status ${ }^{6}$. Prophylactic treatment(s), such as hysterectomy, bilateral mastectomy, salpingectomy, and oophorectomy surgeries remain the gold standard for preventing breast and ovarian cancers among women with these mutations ${ }^{7,8}$, but also recommend ongoing surveillance (e.g., self-examination, magnetic resonance imaging, transvaginal ultrasound, mammogram, etc.) has been recommended biannually ${ }^{9}$.

The impending risk of cancer, the push for prophylactic surgeries, continuous surveillance, and the associated worry of affected family members have been associated with adverse mental health ${ }^{8,10-13}$ and reduced health-related quality of life ${ }^{8,14,15}$. Adverse mental health symptomology is often heightened considering BRCA1/2 diagnoses and what they mean for their health in the future ${ }^{13,16,17}$, often compounded if testing is prompted by a cancer diagnosis ${ }^{18}$. Anxiety and depression are often reported among women with BRCA1/2 mutations, most commonly among those who are undergoing genetic testing ${ }^{19}$, undergoing prophylactic surgeries ${ }^{13}$, and/or during biannual surveillance appointments ${ }^{20}$. Although genetic testing offers preventive opportunities and the knowledge for risk reduction and/or management, it has also been linked to increased anxiety, stress, and depressive symptomology ${ }^{13,21}$, trending at varying levels throughout this process.

Recently, the coronavirus disease 2019 (COVID-19) caused delays in diagnostic investigation, surgical procedures, and routine surveillance for all women, due to limited in-person services ${ }^{22,23}$. Many of these limitations will have long-lasting consequences such as later-stage diagnoses and poorer clinical outcomes ${ }^{24}$, particularly for those with $B R C A 1 / 2$ mutations, who rely on ongoing care for risk reduction and early detection. Individuals with cancer may be at greater risk of COVID-19 complications and death, worsened by older age ( $\geq 60$ years), a history of smoking, obesity, hypertension, cardiovascular disease, and diabetes ${ }^{25-27}$, which are common comorbidities in the general population. The COVID-19 pandemic is expected to have an increase in cancer-related mortality due to care disturbances across the cancer continuum, including but not limited to: 1) reduced access to care due to fear of infection, reallocation of resources, unemployment in the healthcare field, clinic shutdowns; 2) delayed routine care involving preventive screening, abnormal screening and symptom follow-up; 3) later- stage diagnosis indicative of reduced survival, fewer treatment options, and more invasive treatment; and 4) delayed or modified treatments like postponement of treatments and surgeries ${ }^{28}$. Cancer screening during the pandemic decreased $29-36 \%$ from pre-pandemic levels, and specifically, one study found an 86-94\% decline in screening for breast and cervical cancers than 2017-2019 historical averages ${ }^{29}$. The impact of the COVID-19 pandemic on mental health outcomes among high-risk women, a population that already experience high rates of anxiety and depression ${ }^{19,21,30}$, has not yet been investigated.

Objectives. The current study aims to determine the association between several COVID-19 pandemic experiences and anxiety and depression symptomology, while adjusting for covariates among BRCA1/2-positive US women. Secondarily, we stratified these associations by income status and race/ethnicity to identify high-risk groups of mutation carriers. The importance of this paper remains unprecedented, as those with increased risk for breast and ovarian cancer due to genetic mutations may have experienced limited preventive, diagnostic, and/or treatment-related care as the COVID-19 pandemic continues into 2021.

\section{Methods}

\section{Study Design \& Sample}


Participants were recruited through national, online support groups: BRCA1 BRCA2 Genetic Ovarian \& Breast Cancer Gene ( 11,000 members), BRCA Genetic Sisters Support Group ( 6,000 members), BRCA1 \& BRCA2 Support Group ( 3,300 members), BRCA Strong ( 2,500 members), BRCA Sisterhood of Hope ( 1,400 members), Facing Our Risk for Cancer Empowered (FORCE) message boards, Understanding BRCA ( 1,500 members), BRCA Advanced \& Other Hereditary Cancers Journal Club ( 3,200 members), and BRCA Preventive Mastectomy \& Hysterectomy Support Group ( 900 members) from December 2020 to April 2021. One study recruitment post was posted per day within each group (BRCA Strong only allowed one post per week), with written permission obtained from group moderators prior to posting. The post consisted of a brief announcement introducing the study, eligibility criteria, and a link to an anonymous survey. Participants were eligible if they were 18 years or older, female, lived in the US, could read/speak in English, have undergone and tested positive for either (or both) BRCA1 and/or BRCA2 genetic mutations within the past five years, and identify with at least one medically underserved population (i.e., racial, ethnic, and/or sexual minority, person with a physical disability, those with low income, first-generation immigrant, and/or those who are chronically ill). By clicking the brief study announcement, potential participants were rerouted to an anonymous screener survey to determine eligibility, and those fitting criteria were rerouted to the full online survey via REDCap (Research Electronic Data Capture) hosted at the Johns Hopkins Bloomberg School of Public Health $(\mathrm{JHSPH})^{31,32}$. Survey questions prompted participants to rate anxiety, depression, COVID-19 impact, demographic characteristics, clinical cancer and genetic testing information, prophylactic surgery and ongoing surveillance history, body satisfaction, perceived worry of cancer, cancer empowerment, health-related quality of life, discrimination, and healthcare access. Participants who completed the online survey were compensated with a \$20 Amazon e-gift card. This study was approved and conducted according to the ethical standards of the JHSPH Institutional Review Board (IRB) and informed consent was obtained from all participants.

\section{Model Variables}

Predictor variables. To measure the impact of COVID-19, the Pandemic Stress Index ${ }^{33}$ was utilized within the current study. The items involving COVID-19 experiences were as follows: changes in life due to COVID-19, diagnosed with COVID-19, fear of getting or spreading COVID-19, worrying about loved ones, quarantining or isolation, caring for someone at home, working from home, lost job, changes in healthcare services, stigma or discrimination, personal financial loss, frustration/boredom, not having basic supplies, more depression, more anxiety, sleep issues, increased substance use, change in sexual activity, loneliness, confusion about COVID-19, giving to the greater good by following COVID-19 mandates, and getting emotional or financial support from loved ones. The COVID-19 experiential items were entered as predictors, with one predictor in each model. Predictors were originally dichotomous with either "no" did not experience (referent) or "yes" experienced the COVID-19-related prompt during the pandemic. Items ranged from general COVID-19 occurrences (e.g., diagnosed with COVID-19, quarantining, working from home, etc.), health-related prompts (e.g., anxiety, depression, substance use, frustration/boredom, etc.), or resource reallocation (e.g., changing travel plans, financial loss, needing financial support, etc.).

Outcome assessments. To measure anxiety symptomology, the Generalized Anxiety Disorder 7-item (GAD-7) scale (cite). The GAD-7 is a 7-item, 4-point Likert scale prompting, "How often have you been bothered by the following over the past two weeks?" ranging from 0 (not at all sure) to 3 (nearly every day). Responses were added to create a final score which ranged from zero to 21 with clinical cutoffs for mild (zero to 5), moderate (six to 10), and severe anxiety $(11+)^{34}$. The GAD-7 has a sensitivity of $89 \%$ and specificity of $82 \%$, utilized as a screening tool to recommend further evaluation for those scoring in the moderate to severe range ${ }^{34}$. In the general population, the GAD-7 reflects good reliability $(a=0.89)^{35}$ and excellent within the current sample ( $\left.a=0.93\right)$. For the purposes of this study, clinical cutoffs were dichotomized for mild (referent) and moderate/severe anxiety. Moderate/severe anxiety will be discussed as "high anxiety".

Depressive symptomology was measured using the Patient Health Questionnaire (PHQ-9) Depression Assessment ${ }^{36}$. The PHQ-9 is a 9-item, 4-point Likert scale asking, "Over the last 2 weeks, how often have you been bothered by the following problems?" ranging from 0 (not at all) to 3 (nearly every day). Responses were combined to create a total score which ranged from zero to 27 with clinical cutoffs for minimal (zero to four), mild (five to nine), moderate $(10-14)$, moderately severe (15-19), and severe depression $(20-27)^{36}$. The PHQ-9 has been used in the general population, psychiatric populations, and obstetric-gynecologic populations, with an average sensitivity of $88 \%$ and specificity of $88 \%$ for major depression ${ }^{36}$. In the general population, the PHQ-9 has good reliability $(a=0-86-0.89)$ and excellent reliability $(a=0.90)$ within the current sample. Within the current study, clinical cutoffs were dichotomized for minimal/mild (referent) and moderate/moderately severe/severe depression. Moderate/moderately severe/severe depression will be discussed as "more depressive symptoms".

Covariates and stratifications. The following variables were included as covariates across all models: age at survey completion, number of comorbid conditions, years since genetic testing, education, marital status, race/ethnicity, income status, and cancer survivor status (has a history of cancer versus no cancer history). Age at survey completion, number of comorbid conditions (including a past cancer diagnosis) and years since genetic testing were treated as continuous. Polynomial categorical variables were condensed into the following covariates: education (some college or less [referent], college graduate or above), marital status (married/living as married [referent], not married), survivor/control status (no cancer history [referent], cancer survivor), race/ethnicity (non-Hispanic white [referent], racial/ethnic minority), and income status (at least $\$ 40,000$ USD annually per household [referent], below $\$ 40,000$ USD annually per household). In separate models, stratifications by income status and racial/ethnic minority status were included, however, were entered as covariates when not in use as stratifications.

\section{Statistical Methods}

All analyses were performed using Stata statistical software, version $16^{37}$. Frequency and percentages were analyzed to identify missingness; cases that were missing were dropped for that specific model. Missingness is outlined in Table 1. Chi-square tests for categorical variables and independent samples $t$-tests for continuous variables were conducted to determine potential covariates for demographic characteristics of interest. These analyses compared characteristics by income and racial/ethnic minority status) and are included in Table 1. Adjusted odds ratios (aORs) and 95\% confidence intervals (Cls) were calculated with multivariable logistic regression models to measure the association between predictor (COVID-19 experience; one COVID-19 experience per model) and outcomes (anxiety and depression), while adjusting for age at survey completion, number of comorbid conditions, years since genetic testing, 
education, marital status, survivor/control status, race/ethnicity, and income status. To examine the effect of experiences during COVID-19 on anxiety and depressive symptomology by income status and racial/ethnicity among BRCA1/2-positive women, an interaction term was created for COVID-19 experience combined with income status (did/did not experience during COVID-19 x income status) and race/ethnicity (did/did not experience during COVID-19 $\mathrm{x}$ race/ethnicity) within appropriate models. All tests were two-sided and statistical significance was indicated if $p$-values were below 0.05 .

\section{Results \\ Characteristics of the Study Sample}

Description of the study population and characteristics are shown in Table 1. A total of 211 BRCA1/2-positive women, both with and without a history of cancer meeting inclusion criteria were included in the current study. The sample ranged in age from 18 to $75(M=39.5, S D=10.6)$ and most women did not have a history of cancer $(n=138,65.4 \%)$. Most of the current sample was non-Hispanic white (NHW) $(67.2 \%)$, completed a college degree or above (64.5\%), and was married or living as married (62.6\%). Some of the women did report having at least one physical disability (40.8\%) and most reported having more than one comorbid condition including cancer (61.6\%). Most women reported $\geq \$ 40,000 /$ year for their household incomes (77\%) A total of 49 participants identified as being lesbian, gay, bisexual, transgender, queer/questioning, or something else (LGBTQ+). Study characteristics among NHW and racial/ethnic minority women differed significantly. Racial/ethnic minority women were more often 49 years of age or younger $(p=0.011)$, whereas NHW women had reported significantly more comorbid conditions than racial/ethnic minority women $(p=0.027)$. Some demographic characteristics also differed significantly by income status. Women with household incomes $\geq \$ 40,000 /$ year more often reported a college degree or above $(p=<0.001)$ and being married or lived as married $(p=<0.001)$. COVID-19 experiences also differed by income status and racial/ethnic minority status, as depicted in Table 1. 
Table 1

Participant demographic characteristics and disadvantaged health population factors, overall and by income status and racial/ethnic minority status

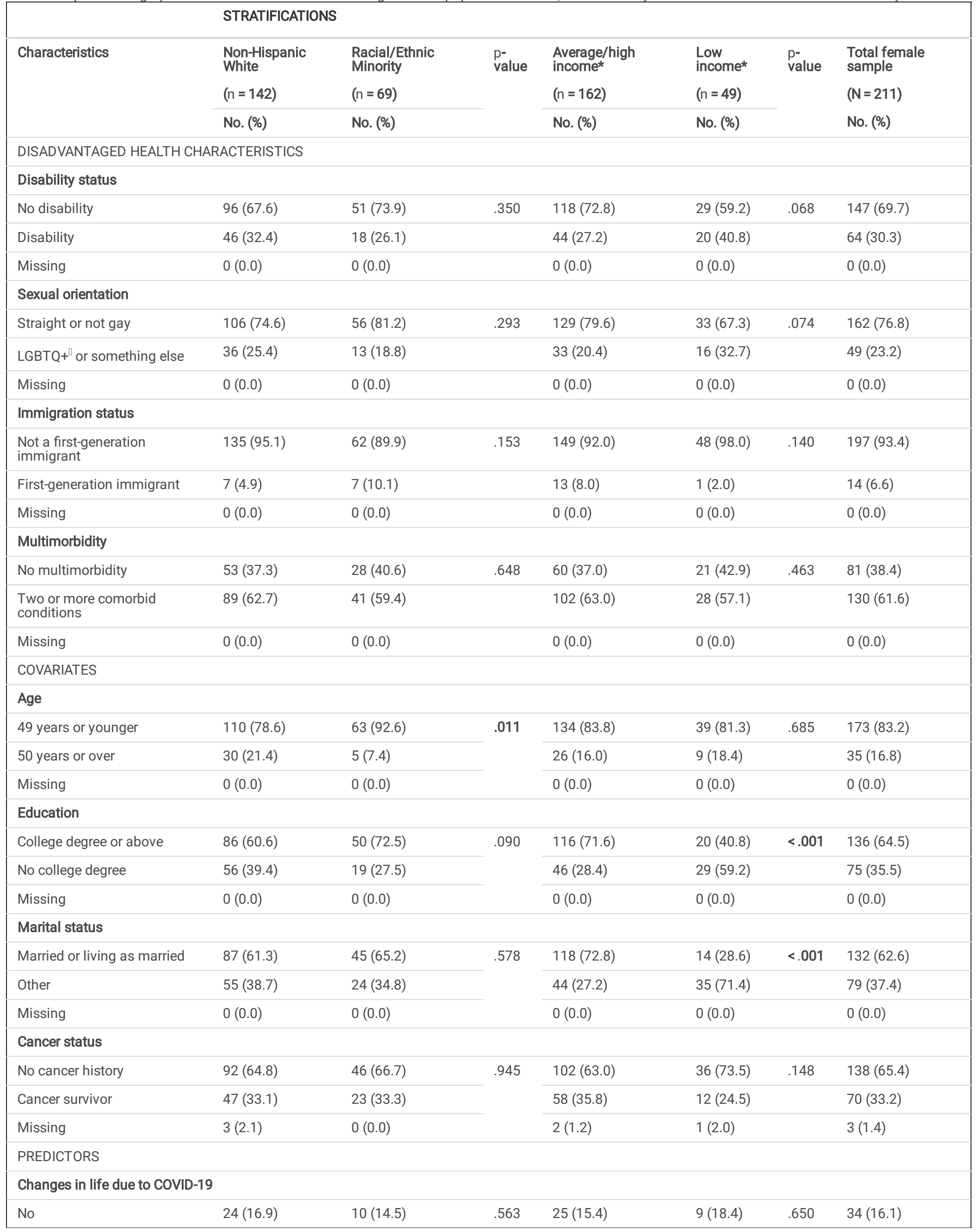




\begin{tabular}{|c|c|c|c|c|c|c|c|}
\hline \multirow[b]{2}{*}{ Yes } & \multicolumn{7}{|c|}{ STRATIFICATIONS } \\
\hline & $106(74.6)$ & $56(81.2)$ & & $125(77.2)$ & $37(75.5)$ & & $162(76.8)$ \\
\hline Missing & $12(8.5)$ & $3(4.3)$ & & $12(7.4)$ & $3(6.1)$ & & $15(7.1)$ \\
\hline \multicolumn{8}{|c|}{ Diagnosed with COVID-19 } \\
\hline No & $131(92.3)$ & $59(85.5)$ & \multirow[t]{3}{*}{.125} & $145(89.5)$ & $45(91.8)$ & .633 & $190(90.0)$ \\
\hline Yes & $11(7.7)$ & $10(14.5)$ & & $17(10.5)$ & $4(8.2)$ & & $21(10.0)$ \\
\hline Missing & $0(0.0)$ & $0(0.0)$ & & $0(0.0)$ & $0(0.0)$ & & $0(0.0)$ \\
\hline \multicolumn{8}{|c|}{ Fear of getting COVID-19 } \\
\hline No & $54(38.0)$ & $30(43.5)$ & \multirow[t]{3}{*}{.448} & $63(38.9)$ & $21(42.9)$ & .619 & $84(39.8)$ \\
\hline Yes & $88(62.0)$ & $39(56.5)$ & & $99(61.1)$ & $28(57.1)$ & & $127(60.2)$ \\
\hline Missing & $0(0.0)$ & $0(0.0)$ & & $0(0.0)$ & $0(0.0)$ & & $0(0.0)$ \\
\hline \multicolumn{8}{|c|}{ Fear of spreading COVID-19 } \\
\hline No & $74(52.1)$ & $47(68.1)$ & \multirow[t]{2}{*}{.027} & $93(57.4)$ & $28(57.1)$ & .974 & $121(57.3)$ \\
\hline Yes & $68(47.9)$ & $22(31.9)$ & & $69(42.6)$ & $21(42.9)$ & & $90(42.7)$ \\
\hline Missing & $0(0.0)$ & $0(0.0)$ & & $0(0.0)$ & $0(0.0)$ & & $0(0.0)$ \\
\hline \multicolumn{8}{|c|}{ Worrying about loved ones } \\
\hline No & $32(22.5)$ & $26(37.7)$ & \multirow[t]{2}{*}{.021} & $50(30.9)$ & $8(16.3)$ & .046 & $58(27.5)$ \\
\hline Yes & $110(77.5)$ & $43(62.3)$ & & $112(69.1)$ & $41(83.7)$ & & $153(72.5)$ \\
\hline Missing & $0(0.0)$ & $0(0.0)$ & & $0(0.0)$ & $0(0.0)$ & & $0(0.0)$ \\
\hline \multicolumn{8}{|c|}{ Quarantining and isolation } \\
\hline No & $54(38.0)$ & $31(44.9)$ & .338 & $70(43.2)$ & $15(30.6)$ & .115 & $85(40.3)$ \\
\hline Yes & $88(62.0)$ & $38(55.1)$ & & $92(56.8)$ & $34(69.4)$ & & $126(59.7)$ \\
\hline Missing & $0(0.0)$ & $0(0.0)$ & & $0(0.0)$ & $0(0.0)$ & & $0(0.0)$ \\
\hline \multicolumn{8}{|c|}{ Caring for someone at home } \\
\hline No & $124(87.3)$ & $56(81.2)$ & .235 & $144(88.9)$ & $36(73.5)$ & .008 & $180(85.3)$ \\
\hline Yes & $18(12.7)$ & $13(18.8)$ & & $18(11.1)$ & $13(26.5)$ & & $31(14.7)$ \\
\hline Missing & $0(0.0)$ & $0(0.0)$ & & $0(0.0)$ & $0(0.0)$ & & $0(0.0)$ \\
\hline \multicolumn{8}{|c|}{ Working from home } \\
\hline No & $88(62.0)$ & $44(63.8)$ & .800 & $95(58.6)$ & $37(75.5)$ & .033 & $132(62.6)$ \\
\hline Yes & $54(38.0)$ & $25(36.2)$ & & $67(41.4)$ & $12(24.5)$ & & $79(37.4)$ \\
\hline Missing & $0(0.0)$ & $0(0.0)$ & & $0(0.0)$ & $0(0.0)$ & & $0(0.0)$ \\
\hline \multicolumn{8}{|c|}{ Lost job due to COVID-19 } \\
\hline No & $129(90.8)$ & $64(92.8)$ & .642 & $153(94.4)$ & $40(81.6)$ & .005 & $193(91.5)$ \\
\hline Yes & $13(9.2)$ & $5(7.2)$ & & $9(5.6)$ & $9(18.4)$ & & $18(8.5)$ \\
\hline Missing & $0(0.0)$ & $0(0.0)$ & & $0(0.0)$ & $0(0.0)$ & & $0(0.0)$ \\
\hline \multicolumn{8}{|c|}{$\begin{array}{l}\text { Changes in healthcare } \\
\text { services }\end{array}$} \\
\hline No & $105(73.9)$ & $51(73.9)$ & .996 & $127(78.4)$ & $29(59.2)$ & .007 & $156(73.9)$ \\
\hline Yes & $37(26.1)$ & $18(26.1)$ & & $35(21.6)$ & $20(40.8)$ & & $55(26.1)$ \\
\hline Missing & $0(0.0)$ & $0(0.0)$ & & $0(0.0)$ & $0(0.0)$ & & $0(0.0)$ \\
\hline \multicolumn{8}{|c|}{ Stigma or discrimination } \\
\hline No & $129(90.8)$ & $64(92.8)$ & .642 & $149(92.0)$ & $44(89.8)$ & .632 & $193(91.5)$ \\
\hline Yes & $13(9.2)$ & $5(7.2)$ & & $13(8.0)$ & $5(10.2)$ & & $18(8.5)$ \\
\hline Missing & $0(0.0)$ & $0(0.0)$ & & $0(0.0)$ & $0(0.0)$ & & $0(0.0)$ \\
\hline
\end{tabular}


STRATIFICATIONS

\section{Personal financial loss}

\begin{tabular}{|c|c|c|c|c|c|c|c|}
\hline No & $97(68.3)$ & $53(76.8)$ & .201 & $126(77.8)$ & $24(49.0)$ & $<.001$ & $150(71.1)$ \\
\hline Yes & $45(31.7)$ & $16(23.2)$ & & $36(22.2)$ & $25(51.0)$ & & $61(28.9)$ \\
\hline Missing & $0(0.0)$ & $0(0.0)$ & & $0(0.0)$ & $0(0.0)$ & & $0(0.0)$ \\
\hline \multicolumn{8}{|c|}{ Frustration or boredom } \\
\hline No & $82(57.7)$ & $35(50.7)$ & .336 & $92(56.8)$ & $25(51.0)$ & .476 & $117(55.5)$ \\
\hline Yes & $60(42.3)$ & $34(49.3)$ & & $70(43.2)$ & $24(49.0)$ & & $94(44.5)$ \\
\hline Missing & $0(0.0)$ & $0(0.0)$ & & $0(0.0)$ & $0(0.0)$ & & $0(0.0)$ \\
\hline \multicolumn{8}{|c|}{ Not having enough basic supplies } \\
\hline No & $130(91.5)$ & $62(89.9)$ & .687 & $150(92.6)$ & $42(85.7)$ & .141 & $192(91.0)$ \\
\hline Yes & $12(8.5)$ & $7(10.1)$ & & $12(7.4)$ & $7(14.3)$ & & $19(9.0)$ \\
\hline Missing & $0(0.0)$ & $0(0.0)$ & & $0(0.0)$ & $0(0.0)$ & & $0(0.0)$ \\
\hline \multicolumn{8}{|c|}{ More anxiety } \\
\hline No & $73(51.4)$ & $32(46.4)$ & .493 & $82(50.6)$ & $23(46.9)$ & .652 & $105(49.8)$ \\
\hline Yes & $69(48.6)$ & $37(53.6)$ & & $80(49.4)$ & $26(53.1)$ & & $106(50.2)$ \\
\hline Missing & $0(0.0)$ & $0(0.0)$ & & $0(0.0)$ & $0(0.0)$ & & $0(0.0)$ \\
\hline \multicolumn{8}{|c|}{ More depression } \\
\hline No & $101(71.1)$ & $40(58.0)$ & .057 & $107(66.0)$ & $34(69.4)$ & .664 & $141(66.8)$ \\
\hline Yes & $41(28.9)$ & $29(42.0)$ & & $55(34.0)$ & $15(30.6)$ & & $70(33.2)$ \\
\hline Missing & $0(0.0)$ & $0(0.0)$ & & $0(0.0)$ & $0(0.0)$ & & $0(0.0)$ \\
\hline \multicolumn{8}{|c|}{ Sleep issues } \\
\hline No & $80(56.3)$ & $39(56.5)$ & .980 & $98(60.5)$ & $21(42.9)$ & .029 & $119(56.4)$ \\
\hline Yes & $62(43.7)$ & $30(43.5)$ & & $64(39.5)$ & $28(57.1)$ & & $92(43.6)$ \\
\hline Missing & $0(0.0)$ & $0(0.0)$ & & $0(0.0)$ & $0(0.0)$ & & $0(0.0)$ \\
\hline \multicolumn{8}{|c|}{ Increased substance use } \\
\hline No & $126(88.7)$ & $59(85.5)$ & .504 & $142(87.7)$ & $43(87.8)$ & .985 & $185(87.7)$ \\
\hline Yes & $16(11.3)$ & $10(14.5)$ & & $20(12.3)$ & $6(12.2)$ & & $26(12.3)$ \\
\hline Missing & $0(0.0)$ & $0(0.0)$ & & $0(0.0)$ & $0(0.0)$ & & $0(0.0)$ \\
\hline \multicolumn{8}{|c|}{ Change in sexual activity } \\
\hline No & $123(86.6)$ & 55 (79.7) & .195 & $134(82.7)$ & $44(89.8)$ & .232 & $178(84.4)$ \\
\hline Yes & $19(13.4)$ & $14(20.3)$ & & $28(17.3)$ & $5(10.2)$ & & $33(15.6)$ \\
\hline Missing & $0(0.0)$ & $0(0.0)$ & & $0(0.0)$ & $0(0.0)$ & & $0(0.0)$ \\
\hline \multicolumn{8}{|c|}{ Loneliness } \\
\hline No & $91(64.1)$ & $37(53.6)$ & .144 & $101(62.3)$ & $27(55.1)$ & .363 & $128(60.7)$ \\
\hline Yes & $51(35.9)$ & $32(46.4)$ & & $61(37.7)$ & $22(44.9)$ & & $83(39.3)$ \\
\hline Missing & $0(0.0)$ & $0(0.0)$ & & $0(0.0)$ & $0(0.0)$ & & $0(0.0)$ \\
\hline \multicolumn{8}{|c|}{ Confusion about COVID-19 } \\
\hline No & $132(93.0)$ & $64(92.8)$ & .957 & $150(92.6)$ & $46(93.9)$ & .759 & $196(92.9)$ \\
\hline Yes & $10(7.0)$ & $5(7.2)$ & & $12(7.4)$ & $3(6.1)$ & & $15(7.1)$ \\
\hline Missing & $0(0.0)$ & $0(0.0)$ & & $0(0.0)$ & $0(0.0)$ & & $0(0.0)$ \\
\hline \multicolumn{8}{|c|}{ Giving to the greater good by following mandates } \\
\hline No & $88(62.0)$ & $56(81.2)$ & .005 & $116(71.6)$ & $28(57.1)$ & .057 & $144(68.2)$ \\
\hline
\end{tabular}




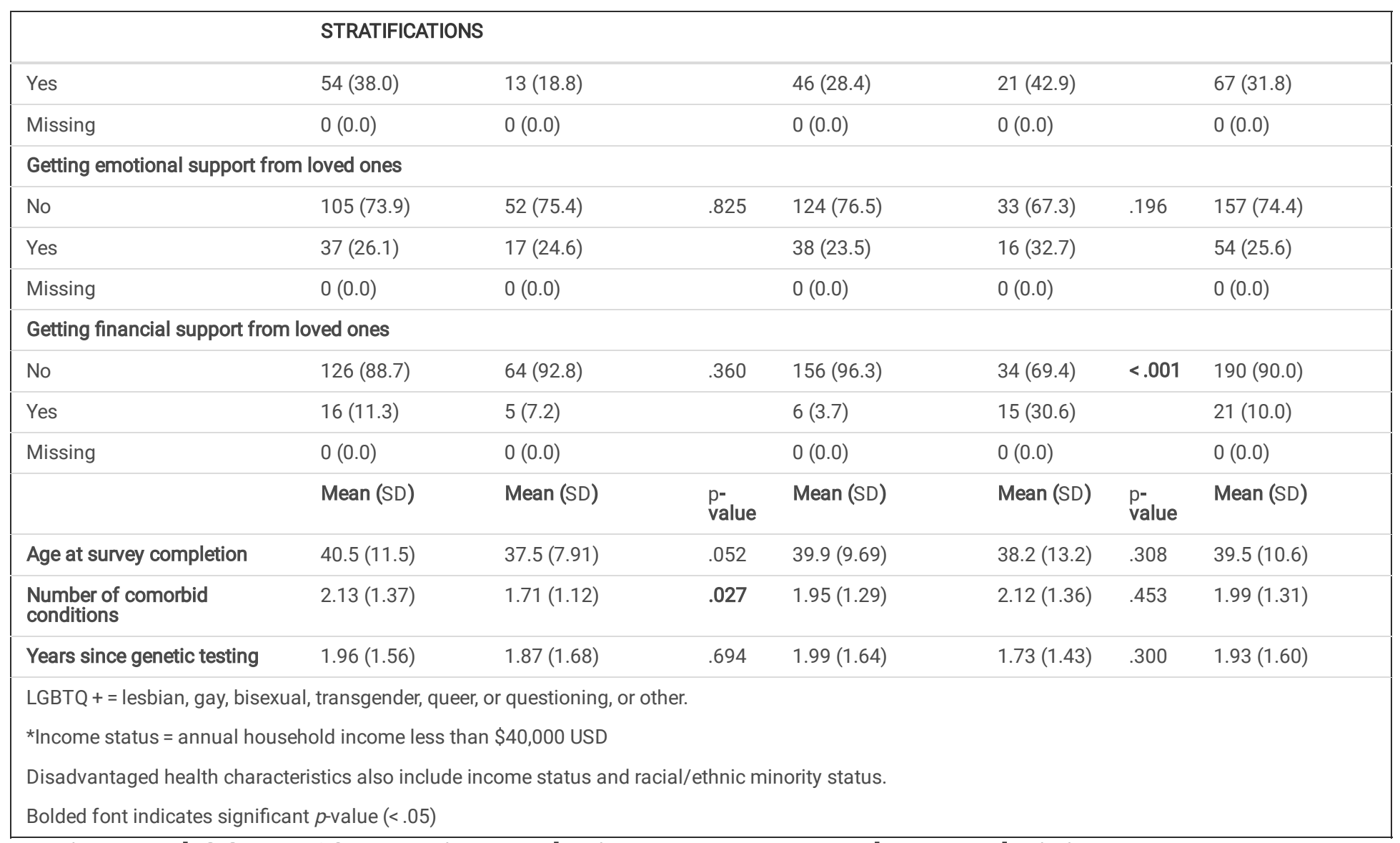

\section{Anxiety and COVID-19 experiences by income status and race/ethnicity}

Table 2 depicts the associations between specific COVID-19 experiences and odds of reporting high anxiety overall and stratified by income status. Overall, women who reported experiencing stigma or discrimination during the COVID-19 pandemic were 4.31 times more likely to also report higher anxiety (95\% $\mathrm{Cl}$, 1.12-16.4) than women who did not experience stigma or discrimination. Similarly, women who reported depression (aOR, 4.82, 2.28-10.1), sleep issues (aOR, 2.31, 95\% Cl, 1.19-4.47), increased substance use (aOR, 2.83, 95\% Cl, 1.03-7.82), or changes in sexual activity (aOR, 2.64, 95\% Cl, 1.03-6.80) due to the COVID-19 pandemic were significantly more likely to report high anxiety than women who did not report these during COVID-19. In stratified analyses, women with low income having trouble finding basic supplies (aOR, 9.32, 95\% Cl, 1.09-79.5), and associations were stronger for depression (aOR, 6.71, 95\% $\mathrm{Cl}, 2.67-$ 16.8), sleep issues (aOR, 3.38, 95\% Cl, 1.54-7.40), and increased substance use (aOR, 4.31, 95\% Cl, 1.14-16.2). These associations were not statistically significant among women with higher income. The only significant stratified interaction was among women with high income who experienced a change in healthcare services due to COVID-19. These women were significantly less likely to report high anxiety $(\mathrm{aOR}, 0.23,95 \% \mathrm{Cl}, 0.06-0.90, p$-interaction $=0.037)$ while women with incomes $<\$ 40,000$ were more likely to report high anxiety $(\mathrm{aOR}, 1.24,95 \% \mathrm{Cl}, 0.53-2.93)$, although this finding was not statistically significant.

Table 2 also shows the relationship between several COVID-19 experiences and the odds of reporting high anxiety overall and stratified by race/ethnicity. Overall, women who experienced stigma or discrimination ( $\mathrm{aOR}, 4.12,95 \% \mathrm{Cl}, 1.06-15.9$ ), not having enough basic supplies (aOR, 3.44, 95\% $\mathrm{Cl}, 1.03-11.4$ ), depression (aOR, 5.33, 95\% Cl, 2.49-11.3), sleep issues (aOR, 2.42, 95\% Cl, 1.24-4.71), increased substance use (aOR, 2.75, 95\% $\mathrm{Cl}, 1.01-7.49$ ), or changes in sexual activity (aOR, $2.57,95 \% \mathrm{Cl}, 1.01-6.57)$ due to the pandemic were significantly more likely to have high anxiety compared to women who did not experience these during the pandemic. Racial/ethnic minority women reporting stigma or discrimination (aOR, 6.94, 95\% $\mathrm{Cl}$, 1.33-35.9) or sleep issues during COVID-19 (aOR, 2.45, 95\% Cl, 1.11-5.40) were more likely to experience high anxiety when compared with racial/ethnic minority women who did not have these experiences. Both NHW (aOR, 7.91, 95\% Cl, 1.87-33.4) and racial/ethnic minority women (aOR, 4.13, 95\% Cl, 1.70-10.0) who experienced depression during the pandemic were more likely to have high anxiety. When stratified, racial/ethnic minority women caring for someone at home during COVID-19 were 3.78 times more likely $(95 \% \mathrm{Cl}, 1.04-13.6)$ to report high anxiety while $\mathrm{NHW}$ women were less likely to have high anxiety with caring for someone at home $(\mathrm{aOR}, 0.36,95 \% \mathrm{Cl}, 0.10-1.33, p$-interaction $=0.011)$. There were two additional interactions: working from home $(p$-interaction $=0.044)$ and experiencing changes in healthcare services ( $p$-interaction $=0.026$ ), but neither resulted in significant odds of high anxiety based on race/ethnicity. 
Table 2

Adjusted odds ratios (aOR) and 95\% confidence intervals $(\mathrm{Cl})$ for the association between COVID-19 experiences and odds of high anxiety among BRCA1/2 women from disadvantaged health populations, overall and by income status and race/ethnicity

\begin{tabular}{|c|c|c|c|c|c|c|c|c|c|c|c|c|c|}
\hline & \multicolumn{2}{|c|}{$\begin{array}{l}\text { All women \& } \\
\text { covariates } \\
(\mathrm{N}=190)\end{array}$} & \multicolumn{2}{|c|}{$\begin{array}{l}\text { Low income } \\
(\mathrm{N}=44)\end{array}$} & \multicolumn{2}{|c|}{$\begin{array}{l}\text { Average/high } \\
\text { income } \\
(\mathrm{N}=146)\end{array}$} & \multirow[t]{2}{*}{ int. } & \multicolumn{2}{|c|}{$\begin{array}{l}\text { All women \& } \\
\text { covariates } \\
(N=190)\end{array}$} & \multicolumn{2}{|c|}{$\begin{array}{l}\text { Racial/ethnic } \\
\text { minority } \\
(\mathrm{N}=64)\end{array}$} & \multicolumn{2}{|c|}{$\begin{array}{l}\text { NHW } \\
(\mathrm{N}=126)\end{array}$} \\
\hline & aOR & $95 \% \mathrm{Cl}$ & aOR & $95 \% \mathrm{Cl}$ & aOR & $95 \% \mathrm{Cl}$ & & aOR & $95 \% \mathrm{Cl}$ & aOR & $95 \% \mathrm{Cl}$ & aOR & $95 \% \mathrm{Cl}$ \\
\hline \multicolumn{14}{|c|}{$\begin{array}{l}\text { Changes in life due to } \\
\text { COVID-19 }\end{array}$} \\
\hline No (33) & 1.00 & reference & 1.00 & reference & 1.00 & reference & .612 & 1.00 & reference & 1.00 & reference & 1.00 & reference \\
\hline Yes (157) & 1.17 & $\begin{array}{l}.511- \\
2.68\end{array}$ & 1.27 & $\begin{array}{l}.490- \\
3.30\end{array}$ & .788 & $\begin{array}{l}.155- \\
3.98\end{array}$ & & 1.21 & $\begin{array}{l}.536- \\
2.75\end{array}$ & 1.33 & $\begin{array}{l}.502- \\
3.52\end{array}$ & .702 & $\begin{array}{l}.129- \\
3.81\end{array}$ \\
\hline
\end{tabular}

\section{Diagnosed with}

COVID-19

\begin{tabular}{|c|c|c|c|c|c|c|c|c|c|c|c|c|c|}
\hline No (169) & 1.00 & reference & 1.00 & reference & 1.00 & reference & .190 & 1.00 & reference & 1.00 & reference & 1.00 & reference \\
\hline Yes (21) & .878 & $\begin{array}{l}.331- \\
2.32\end{array}$ & .624 & $\begin{array}{l}.210- \\
1.84\end{array}$ & 4.01 & $\begin{array}{l}.316- \\
51.1\end{array}$ & & .984 & $\begin{array}{l}.382- \\
2.53\end{array}$ & .930 & $\begin{array}{l}.257- \\
3.35\end{array}$ & .808 & $\begin{array}{l}.177- \\
3.69\end{array}$ \\
\hline
\end{tabular}

Fear of getting COVID-

19

\begin{tabular}{|c|c|c|c|c|c|c|c|c|c|c|c|c|c|}
\hline No (68) & 1.00 & reference & 1.00 & reference & 1.00 & reference & .891 & 1.00 & reference & 1.00 & reference & 1.00 & reference \\
\hline Yes (122) & 1.36 & $\begin{array}{l}.715- \\
2.59\end{array}$ & 1.33 & $\begin{array}{l}.631- \\
2.81\end{array}$ & 1.20 & $\begin{array}{l}.327- \\
4.40\end{array}$ & & 1.22 & $\begin{array}{l}.649- \\
2.31\end{array}$ & 1.76 & $\begin{array}{l}.798- \\
3.89\end{array}$ & .672 & $\begin{array}{l}.207- \\
2.17\end{array}$ \\
\hline
\end{tabular}

Fear of spreading

COVID-19

\begin{tabular}{|c|c|c|c|c|c|c|c|c|c|c|c|c|c|}
\hline No (103) & 1.00 & reference & 1.00 & reference & 1.00 & reference & .767 & 1.00 & reference & 1.00 & reference & 1.00 & reference \\
\hline Yes (87) & 1.21 & $\begin{array}{l}.647- \\
2.27\end{array}$ & 1.26 & $\begin{array}{l}.619- \\
2.59\end{array}$ & 1.01 & $\begin{array}{l}.272- \\
3.76\end{array}$ & & 1.01 & $\begin{array}{l}.551- \\
1.86\end{array}$ & 1.34 & $\begin{array}{l}.638- \\
2.83\end{array}$ & .907 & $\begin{array}{l}.275- \\
2.98\end{array}$ \\
\hline
\end{tabular}

Worrying about loved

ones

\begin{tabular}{|c|c|c|c|c|c|c|c|c|c|c|c|c|c|}
\hline No (42) & 1.00 & reference & 1.00 & reference & 1.00 & reference & .265 & 1.00 & reference & 1.00 & reference & 1.00 & reference \\
\hline Yes (148) & 1.23 & $\begin{array}{l}.575- \\
2.66\end{array}$ & 1.06 & $\begin{array}{l}.461- \\
2.46\end{array}$ & 4.45 & $\begin{array}{l}.409- \\
48.4\end{array}$ & & 1.06 & $\begin{array}{l}.507- \\
2.23\end{array}$ & 1.43 & $\begin{array}{l}.514- \\
4.01\end{array}$ & 1.08 & $\begin{array}{l}.324- \\
3.65\end{array}$ \\
\hline \multicolumn{14}{|c|}{ Quarantining/Isolation } \\
\hline No (67) & 1.00 & reference & 1.00 & reference & 1.00 & reference & .192 & 1.00 & reference & 1.00 & reference & 1.00 & reference \\
\hline Yes (123) & .869 & $\begin{array}{l}.458- \\
1.65\end{array}$ & 1.10 & $\begin{array}{l}.538- \\
2.27\end{array}$ & .373 & $\begin{array}{l}.086- \\
1.61\end{array}$ & & .831 & $\begin{array}{l}.443- \\
1.56\end{array}$ & .918 & $\begin{array}{l}.417- \\
2.02\end{array}$ & .819 & $\begin{array}{l}.259- \\
2.58\end{array}$ \\
\hline
\end{tabular}

\section{Caring for someone at}

home

$\begin{array}{llllllllllllll}\text { No (162) } & 1.00 & \text { reference } & 1.00 & \text { reference } & 1.00 & \text { reference } & .418 & 1.00 & \text { reference } & 1.00 & \text { reference } & 1.00 & \text { reference } \\ \text { Yes (28) } & 1.19 & .488- & .992 & .316- & 2.18 & .479- & & 1.53 & .629- & \mathbf{3 . 7 8} & \mathbf{1 . 0 4 -} & .359 & .096- \\ & & 2.94 & & 3.11 & & 10.0 & & & 3.73 & & 13.6 & \end{array}$

Working from home

\begin{tabular}{|c|c|c|c|c|c|c|c|c|c|c|c|c|c|}
\hline No (112) & 1.00 & reference & 1.00 & reference & 1.00 & reference & .119 & 1.00 & reference & 1.00 & reference & 1.00 & reference \\
\hline Yes (78) & 1.10 & $\begin{array}{l}.576- \\
2.12\end{array}$ & 1.35 & $\begin{array}{l}.638- \\
2.89\end{array}$ & .354 & $\begin{array}{l}.079- \\
1.58\end{array}$ & & .962 & $\begin{array}{l}.503- \\
1.84\end{array}$ & 1.56 & $\begin{array}{l}.713- \\
3.41\end{array}$ & .384 & $\begin{array}{l}.120- \\
1.23\end{array}$ \\
\hline
\end{tabular}

Lost job due to COVID-

19

\begin{tabular}{|c|c|c|c|c|c|c|c|c|c|c|c|c|c|}
\hline No (172) & 1.00 & reference & 1.00 & reference & 1.00 & reference & .580 & 1.00 & reference & 1.00 & reference & 1.00 & reference \\
\hline Yes (18) & .809 & $\begin{array}{l}.285- \\
2.29\end{array}$ & 1.24 & $\begin{array}{l}.282- \\
5.45\end{array}$ & .662 & $\begin{array}{l}.126- \\
3.47\end{array}$ & & .921 & $\begin{array}{l}.321- \\
2.64\end{array}$ & .745 & $\begin{array}{l}.211- \\
2.63\end{array}$ & 1.89 & $\begin{array}{l}.185- \\
19.2\end{array}$ \\
\hline
\end{tabular}

Change in healthcare

services

\begin{tabular}{|c|c|c|c|c|c|c|c|c|c|c|c|c|c|}
\hline No (135) & 1.00 & reference & 1.00 & reference & 1.00 & reference & .037 & 1.00 & reference & 1.00 & reference & 1.00 & reference \\
\hline Yes (55) & .695 & $\begin{array}{l}.350- \\
1.38\end{array}$ & 1.24 & $\begin{array}{l}.530- \\
2.93\end{array}$ & .234 & $\begin{array}{l}.060 \\
-.903\end{array}$ & & .794 & $\begin{array}{l}.397- \\
1.58\end{array}$ & .431 & $\begin{array}{l}.178- \\
1.04\end{array}$ & 2.88 & $\begin{array}{l}.691- \\
12.0\end{array}$ \\
\hline
\end{tabular}




\begin{tabular}{|c|c|c|c|c|c|c|c|c|c|c|c|c|c|}
\hline & \multicolumn{2}{|c|}{$\begin{array}{l}\text { All women \& } \\
\text { covariates } \\
(N=190)\end{array}$} & \multicolumn{2}{|c|}{$\begin{array}{l}\text { Low income } \\
(\mathrm{N}=44)\end{array}$} & \multicolumn{2}{|c|}{$\begin{array}{l}\text { Average/high } \\
\text { income } \\
(\mathrm{N}=146)\end{array}$} & \multirow[t]{2}{*}{ int. } & \multicolumn{2}{|c|}{$\begin{array}{l}\text { All women \& } \\
\text { covariates } \\
(\mathrm{N}=190)\end{array}$} & \multicolumn{2}{|c|}{$\begin{array}{l}\text { Racial/ethnic } \\
\text { minority } \\
(\mathrm{N}=64)\end{array}$} & \multicolumn{2}{|c|}{$\begin{array}{l}\text { NHW } \\
(N=126)\end{array}$} \\
\hline & aOR & $95 \% \mathrm{Cl}$ & $\mathrm{aOR}$ & $95 \% \mathrm{Cl}$ & $\mathrm{aOR}$ & $95 \% \mathrm{Cl}$ & & aOR & $95 \% \mathrm{Cl}$ & aOR & $95 \% \mathrm{Cl}$ & aOR & $95 \% \mathrm{Cl}$ \\
\hline \multicolumn{14}{|c|}{$\begin{array}{l}\text { Stigma or } \\
\text { discrimination }\end{array}$} \\
\hline No (172) & 1.00 & reference & 1.00 & reference & 1.00 & reference & .621 & 1.00 & reference & 1.00 & reference & 1.00 & reference \\
\hline Yes (18) & 4.31 & $\begin{array}{l}1.12- \\
16.4\end{array}$ & 3.58 & $\begin{array}{l}.701- \\
18.3\end{array}$ & 7.65 & $\begin{array}{l}.639- \\
91.6\end{array}$ & & 4.12 & $\begin{array}{l}1.06- \\
15.9\end{array}$ & 6.94 & $\begin{array}{l}1.33- \\
35.9\end{array}$ & 1.33 & $\begin{array}{l}.134- \\
13.2\end{array}$ \\
\hline \multicolumn{14}{|c|}{ Personal financial loss } \\
\hline No (129) & 1.00 & reference & 1.00 & reference & 1.00 & reference & .475 & 1.00 & reference & 1.00 & reference & 1.00 & reference \\
\hline Yes (61) & .800 & $\begin{array}{l}.414- \\
1.54\end{array}$ & 1.07 & $\begin{array}{l}.476- \\
2.41\end{array}$ & .616 & $\begin{array}{l}.169- \\
2.23\end{array}$ & & .847 & $\begin{array}{l}.432- \\
1.66\end{array}$ & .905 & $\begin{array}{l}.401- \\
2.03\end{array}$ & .947 & $\begin{array}{l}.268- \\
3.34\end{array}$ \\
\hline \multicolumn{14}{|c|}{ Frustration/Boredom } \\
\hline No (99) & 1.00 & reference & 1.00 & reference & 1.00 & reference & .574 & 1.00 & reference & 1.00 & reference & 1.00 & reference \\
\hline Yes (91) & 1.05 & $\begin{array}{l}.558- \\
2.00\end{array}$ & 1.20 & $\begin{array}{l}.585- \\
2.47\end{array}$ & .792 & $\begin{array}{l}.213- \\
2.94\end{array}$ & & 1.16 & $\begin{array}{l}.617- \\
2.19\end{array}$ & 1.05 & $\begin{array}{l}.493- \\
2.24\end{array}$ & 1.21 & $\begin{array}{l}.382- \\
3.85\end{array}$ \\
\hline \multicolumn{14}{|c|}{$\begin{array}{l}\text { Not having basic } \\
\text { supplies }\end{array}$} \\
\hline No (171) & 1.00 & reference & 1.00 & reference & 1.00 & reference & .186 & 1.00 & reference & 1.00 & reference & 1.00 & reference \\
\hline Yes (19) & 3.15 & $\begin{array}{l}.943- \\
10.5\end{array}$ & 9.32 & $\begin{array}{l}1.09- \\
79.5\end{array}$ & 1.38 & $\begin{array}{l}.230- \\
8.34\end{array}$ & & 3.44 & $\begin{array}{l}1.03- \\
11.4\end{array}$ & 2.54 & $\begin{array}{l}.664- \\
9.73\end{array}$ & - & - \\
\hline \multicolumn{14}{|c|}{ More depression } \\
\hline No (123) & 1.00 & reference & 1.00 & reference & 1.00 & reference & .197 & 1.00 & reference & 1.00 & reference & 1.00 & reference \\
\hline Yes (67) & 4.82 & $\begin{array}{l}2.28- \\
10.1\end{array}$ & 6.71 & $\begin{array}{l}2.67- \\
16.8\end{array}$ & 2.22 & $\begin{array}{l}.533- \\
9.29\end{array}$ & & 5.33 & $\begin{array}{l}2.49- \\
11.3\end{array}$ & 4.13 & $\begin{array}{l}1.70- \\
10.0\end{array}$ & 7.91 & $\begin{array}{l}1.87- \\
33.4\end{array}$ \\
\hline \multicolumn{14}{|c|}{ Sleep issues } \\
\hline No (101) & 1.00 & reference & 1.00 & reference & 1.00 & reference & .146 & 1.00 & reference & 1.00 & reference & 1.00 & reference \\
\hline Yes (89) & 2.31 & $\begin{array}{l}1.19- \\
4.47\end{array}$ & 3.38 & $\begin{array}{l}1.54- \\
7.40\end{array}$ & 1.07 & $\begin{array}{l}.276- \\
4.18\end{array}$ & & 2.42 & $\begin{array}{l}1.24- \\
4.71\end{array}$ & 2.45 & $\begin{array}{l}1.11- \\
5.40\end{array}$ & 3.00 & $\begin{array}{l}.879- \\
10.2\end{array}$ \\
\hline \multicolumn{14}{|c|}{$\begin{array}{l}\text { Increased substance } \\
\text { use }\end{array}$} \\
\hline No (164) & 1.00 & reference & 1.00 & reference & 1.00 & reference & .258 & 1.00 & reference & 1.00 & reference & 1.00 & reference \\
\hline Yes (26) & 2.83 & $\begin{array}{l}1.03- \\
7.82\end{array}$ & 4.31 & $\begin{array}{l}1.14- \\
16.2\end{array}$ & 1.19 & $\begin{array}{l}.192- \\
7.35\end{array}$ & & 2.75 & $\begin{array}{l}1.01- \\
7.49\end{array}$ & 2.61 & $\begin{array}{l}.806- \\
8.49\end{array}$ & 3.84 & $\begin{array}{l}.443- \\
33.4\end{array}$ \\
\hline \multicolumn{14}{|c|}{$\begin{array}{l}\text { Change in sexual } \\
\text { activity }\end{array}$} \\
\hline No (158) & 1.00 & reference & 1.00 & reference & 1.00 & reference & .524 & 1.00 & reference & 1.00 & reference & 1.00 & reference \\
\hline Yes (32) & 2.64 & $\begin{array}{l}1.03- \\
6.80\end{array}$ & 3.00 & $\begin{array}{l}1.00- \\
8.93\end{array}$ & 1.43 & $\begin{array}{l}.193- \\
10.6\end{array}$ & & 2.57 & $\begin{array}{l}1.01- \\
6.57\end{array}$ & 1.79 & $\begin{array}{l}.588- \\
5.46\end{array}$ & 6.57 & $\begin{array}{l}.766- \\
56.3\end{array}$ \\
\hline \multicolumn{14}{|c|}{ Loneliness } \\
\hline No (108) & 1.00 & reference & 1.00 & reference & 1.00 & reference & .913 & 1.00 & reference & 1.00 & reference & 1.00 & reference \\
\hline Yes (82) & .901 & $\begin{array}{l}.479- \\
1.69\end{array}$ & .940 & $\begin{array}{l}.455- \\
1.94\end{array}$ & .867 & $\begin{array}{l}.242- \\
3.09\end{array}$ & & .996 & $\begin{array}{l}.533- \\
1.86\end{array}$ & 1.02 & $\begin{array}{l}.475- \\
2.19\end{array}$ & .733 & $\begin{array}{l}.234- \\
2.29\end{array}$ \\
\hline \multicolumn{14}{|c|}{$\begin{array}{l}\text { Confusion about } \\
\text { COVID-19 }\end{array}$} \\
\hline No (175) & 1.00 & reference & 1.00 & reference & 1.00 & reference & .243 & 1.00 & reference & 1.00 & reference & 1.00 & reference \\
\hline Yes (15) & 1.91 & $\begin{array}{l}.554- \\
6.64\end{array}$ & 3.52 & $\begin{array}{l}.648- \\
16.3\end{array}$ & .494 & $\begin{array}{l}.032- \\
7.46\end{array}$ & & 1.86 & $\begin{array}{l}.544- \\
6.41\end{array}$ & 2.20 & $\begin{array}{l}.493- \\
9.83\end{array}$ & 1.57 & $\begin{array}{l}.158- \\
15.6\end{array}$ \\
\hline \multicolumn{14}{|c|}{$\begin{array}{l}\text { Giving to greater good } \\
\text { by following } \\
\text { mandates }\end{array}$} \\
\hline No (124) & 1.00 & reference & 1.00 & reference & 1.00 & reference & .568 & 1.00 & reference & 1.00 & reference & 1.00 & reference \\
\hline
\end{tabular}




\begin{tabular}{|c|c|c|c|c|c|c|c|c|c|c|c|c|c|}
\hline & \multicolumn{2}{|c|}{$\begin{array}{l}\text { All women \& } \\
\text { covariates } \\
(\mathrm{N}=190)\end{array}$} & \multicolumn{2}{|c|}{$\begin{array}{l}\text { Low income } \\
(\mathrm{N}=44)\end{array}$} & \multicolumn{2}{|c|}{$\begin{array}{l}\text { Average/high } \\
\text { income } \\
(\mathrm{N}=146)\end{array}$} & \multirow[t]{2}{*}{ int. } & \multicolumn{2}{|c|}{$\begin{array}{l}\text { All women \& } \\
\text { covariates } \\
(\mathrm{N}=190)\end{array}$} & \multicolumn{2}{|c|}{$\begin{array}{l}\text { Racial/ethnic } \\
\text { minority } \\
(\mathrm{N}=64)\end{array}$} & \multicolumn{2}{|c|}{$\begin{array}{l}\text { NHW } \\
(\mathrm{N}=126)\end{array}$} \\
\hline & aOR & $95 \% \mathrm{Cl}$ & aOR & $95 \% \mathrm{Cl}$ & aOR & $95 \% \mathrm{Cl}$ & & aOR & $95 \% \mathrm{Cl}$ & aOR & $95 \% \mathrm{Cl}$ & aOR & $95 \% \mathrm{Cl}$ \\
\hline Yes (66) & .708 & $\begin{array}{l}.368^{-} \\
1.35\end{array}$ & .841 & $\begin{array}{l}.390- \\
1.81\end{array}$ & .546 & $\begin{array}{l}.150- \\
1.97\end{array}$ & & .644 & $\begin{array}{l}.338- \\
1.22\end{array}$ & .696 & $\begin{array}{l}.329- \\
1.46\end{array}$ & .992 & $\begin{array}{l}.241- \\
4.07\end{array}$ \\
\hline
\end{tabular}

Getting emotional

support from loved

ones

$\begin{array}{llllllllllllll}\text { No (138) } & 1.00 & \text { reference } & 1.00 & \text { reference } & 1.00 & \text { reference } & .200 & 1.00 & \text { reference } & 1.00 & \text { reference } & 1.00 & \text { reference } \\ \text { Yes (52) } & 1.27 & .634- & 1.76 & .755- & .626 & .161- & & 1.31 & .650- & 1.53 & .672- & .905 & .246- \\ & & 2.57 & & 4.14 & & 2.43 & & & 2.64 & 3.48 & & 3.32 & \end{array}$

Getting financial

support from loved

ones

\begin{tabular}{|c|c|c|c|c|c|c|c|c|c|c|c|c|c|}
\hline No (169) & 1.00 & reference & 1.00 & reference & 1.00 & reference & .134 & 1.00 & reference & 1.00 & reference & 1.00 & reference \\
\hline Yes (21) & .509 & $\begin{array}{l}.177- \\
1.46\end{array}$ & 1.85 & $\begin{array}{l}.300- \\
11.4\end{array}$ & .319 & $\begin{array}{l}.076- \\
1.32\end{array}$ & & .604 & $\begin{array}{l}.207- \\
1.75\end{array}$ & .889 & $\begin{array}{l}.268- \\
2.93\end{array}$ & .217 & $\begin{array}{l}.029- \\
1.62\end{array}$ \\
\hline
\end{tabular}

Missing values: anxiety (15), change in life due to COVID-19 (15), age (3), and cancer status (3).

Bold font indicates statistically significant with corresponding $p<.05$.

p-interaction terms are between income status and predictor(s).

Covariates/stratifications: age (continuous), number of comorbid conditions (continuous), years since genetic testing (continuous), education (some college college graduate or above), marital status (married/living as married, other), cancer status (no cancer history, cancer history), income status (average/high in income), and race/ethnicity (non-Hispanic white [NHW], Hispanic or racial minority).

\section{Depression and COVID-19 experiences by income status and race/ethnicity}

Table 3 presents the association between many COVID-19-related events and the odds of reporting more depressive symptoms, both overall and by income status. Women reporting stigma or discrimination ( $\mathrm{aOR}, 4.65,95 \% \mathrm{Cl}, 1.45-14.8$ ) or sleep issues (aOR, 2.07, 95\% $\mathrm{Cl}, 1.05-4.06)$ during the COVID-19 pandemic were significantly more likely to have more symptoms of depression than women who did not experience these. Similar to the other models, women with low income reporting stigma or discrimination ( $\mathrm{aOR}, 5.61,95 \% \mathrm{Cl}, 1.28-24.5)$, anxiety $(\mathrm{aOR}, 2.15,95 \% \mathrm{Cl}, 1.02-4.56)$, sleep issues (aOR, 2.95, 95\% $\mathrm{Cl}$, $1.36-6.36)$, increased substance use $(\mathrm{aOR}, 3.28,95 \% \mathrm{Cl}, 1.12-9.61)$, or loneliness ( $\mathrm{aOR}, 2.31,95 \% \mathrm{Cl}, 1.10-4.83$ ) during the pandemic were significantly more likely to report increased depressive symptoms in comparison with women with low income who reported these instances (stigma/discrimination: aOR, 4.65 , 95\% Cl, 1.45-14.8; anxiety: aOR, 1.73, 95\% OR, 0.89-3.36; sleep issues: aOR, 2.07, 95\% Cl, 1.05-4.06; increased substance use: aOR, 2.13, 95\% Cl, 0.86-3.36; loneliness: $\mathrm{aOR}, 1.85,95 \% \mathrm{Cl}, 0.97-3.53)$. There was a significant interaction between women who reported quarantining/isolation and low income status $(p=$ 0.018), where women with higher incomes were significantly less likely to have more depressive symptoms $(\mathrm{aOR}, 0.08,95 \% \mathrm{Cl}, 0.02-0.42)$ than women with lower incomes (aOR, 0.72, 95\% Cl, 0.34-1.50).

Table 3 also depicts the relationship between COVID-19-related encounters and the odds of reporting more depressive symptomology overall and by race/ethnicity. Differing from previous models, women who reported quarantining/isolation (aOR, $0.46,95 \% \mathrm{Cl}, 0.24-0.88)$ and those who followed mandates for the greater good $(\mathrm{aOR}, 0.46,95 \% \mathrm{Cl}, 0.23-0.91)$ experienced significantly fewer depressive symptoms than women who did not report these instances during COVID-19. Like the anxiety models outlined above, women who reported stigma or discrimination (aOR, 4.22, 95\% Cl, 1.35-13.2) due to the pandemic were more likely to report depressive symptoms, with racial/ethnic minorities having 5.95 times the odds (95\% $\mathrm{Cl}, 1.55-22.8)$ and $\mathrm{NHW}$ women having 2.80 times the odds $(95 \% \mathrm{Cl}, 0.28-27.7)$, although this was not statistically significant. Women who experienced sleep issues (aOR, $2.10,95 \% \mathrm{Cl}, 1.07-4.09)$ or loneliness $(\mathrm{aOR}, 2.07,95 \% \mathrm{Cl}, 1.09-3.94)$ during this time were also more likely to report depressive symptomology than women who did not experience them. Interestingly, NHW women who used substances more often during COVID-19 were almost 10 times more likely (aOR, 9.44, 95\% Cl, 1.09-82.5) to report increased depressive symptomology, while the association was $1.13(95 \% \mathrm{Cl}, 0.34-3.75)$ among racial/ethnic minority women. The association between sleep issues due to the pandemic and depression symptoms was significantly modified by race/ethnicity. NHW women reporting sleep issues during the pandemic were 7.39 more likely $(95 \% \mathrm{Cl}, 2.21-24.6)$ to experience more depressive symptoms while minority women were only 1.32 times more likely $(95 \% \mathrm{Cl}$, $0.57-$ 3.01), although this finding was not statistically significant. There were also racial/ethnic differences among women caring for someone at home during the COVID-19 pandemic and odds of depression. NHW women were significantly less likely (aOR, $0.25,95 \% \mathrm{Cl}, 0.06-0.99)$ to report more depressive symptoms, while racial/ethnic minority women were 3.62 times more likely $(95 \% \mathrm{Cl}, 1.15-11.4)$ to experience depression symptomology $(p$-interaction $=0.003)$. 
Table 3

Adjusted odds ratios (OR, aOR) and 95\% confidence intervals $(\mathrm{Cl})$ for the association between COVID-19 experiences and odds of more depressive symptoms a BRCA1/2-positive women from disadvantaged health populations, overall and by income status and race/ethnicity

\begin{tabular}{|c|c|c|c|c|c|c|c|c|c|c|c|c|c|}
\hline & \multicolumn{2}{|c|}{$\begin{array}{l}\text { All women } \\
(\mathrm{N}=194)\end{array}$} & \multicolumn{2}{|c|}{$\begin{array}{l}\text { Low income } \\
(\mathrm{N}=45)\end{array}$} & \multicolumn{2}{|c|}{$\begin{array}{l}\text { Average/high } \\
\text { income } \\
(\mathrm{N}=149)\end{array}$} & \multirow[t]{2}{*}{ int. } & \multicolumn{2}{|c|}{$\begin{array}{l}\text { All women } \\
(N=194)\end{array}$} & \multicolumn{2}{|c|}{$\begin{array}{l}\text { Racial/ethnic } \\
\text { minority } \\
(\mathrm{N}=65)\end{array}$} & \multicolumn{2}{|c|}{$\begin{array}{l}\text { NHW } \\
(\mathrm{N}=129)\end{array}$} \\
\hline & aOR & $95 \% \mathrm{Cl}$ & aOR & $95 \% \mathrm{Cl}$ & aOR & $95 \% \mathrm{Cl}$ & & aOR & $95 \% \mathrm{Cl}$ & aOR & $95 \% \mathrm{Cl}$ & aOR & $95 \% \mathrm{Cl}$ \\
\hline \multicolumn{14}{|c|}{$\begin{array}{l}\text { Changes in life due to } \\
\text { COVID-19 }\end{array}$} \\
\hline No (34) & 1.00 & reference & 1.00 & reference & 1.00 & reference & .333 & 1.00 & reference & 1.00 & reference & 1.00 & reference \\
\hline Yes (160) & .896 & $\begin{array}{l}.387- \\
2.07\end{array}$ & 1.06 & $\begin{array}{l}.407- \\
2.77\end{array}$ & .417 & $\begin{array}{l}.078- \\
2.22\end{array}$ & & .954 & $\begin{array}{l}.419- \\
2.17\end{array}$ & .793 & $\begin{array}{l}.288- \\
2.18\end{array}$ & .996 & $\begin{array}{l}.235- \\
4.22\end{array}$ \\
\hline \multicolumn{14}{|c|}{$\begin{array}{l}\text { Diagnosed with } \\
\text { COVID-19 }\end{array}$} \\
\hline No (173) & 1.00 & reference & 1.00 & reference & 1.00 & reference & .144 & 1.00 & reference & 1.00 & reference & 1.00 & reference \\
\hline Yes (21) & 1.36 & $\begin{array}{l}.506- \\
3.69\end{array}$ & .929 & $\begin{array}{l}.304- \\
2.83\end{array}$ & 8.01 & $\begin{array}{l}.570- \\
112.5\end{array}$ & & 1.56 & $\begin{array}{l}.609- \\
4.02\end{array}$ & 1.69 & $\begin{array}{l}.442- \\
6.52\end{array}$ & 1.06 & $\begin{array}{l}.254- \\
4.46\end{array}$ \\
\hline \multicolumn{14}{|c|}{$\begin{array}{l}\text { Fear of getting COVID- } \\
19\end{array}$} \\
\hline No (69) & 1.00 & reference & 1.00 & reference & 1.00 & reference & .821 & 1.00 & reference & 1.00 & reference & 1.00 & reference \\
\hline Yes (125) & .828 & $\begin{array}{l}.433- \\
1.58\end{array}$ & .810 & $\begin{array}{l}.383- \\
1.70\end{array}$ & .673 & $\begin{array}{l}.165- \\
2.75\end{array}$ & & .717 & $\begin{array}{l}.379- \\
1.35\end{array}$ & .452 & $\begin{array}{l}.194- \\
1.05\end{array}$ & 1.84 & $\begin{array}{l}.636- \\
5.36\end{array}$ \\
\hline \multicolumn{14}{|c|}{$\begin{array}{l}\text { Fear of spreading } \\
\text { COVID-19 }\end{array}$} \\
\hline No (105) & 1.00 & reference & 1.00 & reference & 1.00 & reference & .859 & 1.00 & reference & 1.00 & reference & 1.00 & reference \\
\hline Yes (89) & .686 & $\begin{array}{l}.360- \\
1.30\end{array}$ & .689 & $\begin{array}{l}.335- \\
1.41\end{array}$ & .597 & $\begin{array}{l}.142- \\
2.50\end{array}$ & & .563 & $\begin{array}{l}.301- \\
1.05\end{array}$ & .585 & $\begin{array}{l}.260- \\
1.31\end{array}$ & .863 & $\begin{array}{l}.286- \\
2.60\end{array}$ \\
\hline \multicolumn{14}{|c|}{$\begin{array}{l}\text { Worrying about loved } \\
\text { ones }\end{array}$} \\
\hline No (43) & 1.00 & reference & 1.00 & reference & 1.00 & reference & .793 & 1.00 & reference & 1.00 & reference & 1.00 & reference \\
\hline Yes (151) & .976 & $\begin{array}{l}.453- \\
2.10\end{array}$ & .993 & $\begin{array}{l}.433- \\
2.27\end{array}$ & 1.39 & $\begin{array}{l}.126- \\
15.3\end{array}$ & & .809 & $\begin{array}{l}.387- \\
1.69\end{array}$ & .563 & $\begin{array}{l}.192- \\
1.64\end{array}$ & 1.92 & $\begin{array}{l}.632- \\
5.82\end{array}$ \\
\hline \multicolumn{14}{|c|}{ Quarantining/Isolation } \\
\hline No (69) & 1.00 & reference & 1.00 & reference & 1.00 & reference & .018 & 1.00 & reference & 1.00 & reference & 1.00 & reference \\
\hline Yes (125) & .481 & $\begin{array}{l}.249 \\
-.928\end{array}$ & .724 & $\begin{array}{l}.348- \\
1.50\end{array}$ & .082 & $\begin{array}{l}.015 \\
-.426\end{array}$ & & .467 & $\begin{array}{l}.245 \\
-.887\end{array}$ & .438 & $\begin{array}{l}.189- \\
1.01\end{array}$ & .576 & $\begin{array}{l}.195- \\
1.70\end{array}$ \\
\hline \multicolumn{14}{|c|}{$\begin{array}{l}\text { Caring for someone at } \\
\text { home }\end{array}$} \\
\hline No (164) & 1.00 & reference & 1.00 & reference & 1.00 & reference & .226 & 1.00 & reference & 1.00 & reference & 1.00 & reference \\
\hline Yes (30) & .978 & $\begin{array}{l}.408- \\
2.34\end{array}$ & 1.71 & $\begin{array}{l}.559- \\
5.24\end{array}$ & .501 & $\begin{array}{l}.098- \\
2.54\end{array}$ & & 1.32 & $\begin{array}{l}.561- \\
3.14\end{array}$ & 3.62 & $\begin{array}{l}1.15- \\
11.4\end{array}$ & .251 & $\begin{array}{l}.063 \\
-.997\end{array}$ \\
\hline \multicolumn{14}{|c|}{ Working from home } \\
\hline No (115) & 1.00 & reference & 1.00 & reference & 1.00 & reference & .210 & 1.00 & reference & 1.00 & reference & 1.00 & reference \\
\hline Yes (79) & 1.02 & $\begin{array}{l}.528- \\
1.97\end{array}$ & 1.14 & $\begin{array}{l}.540- \\
2.44\end{array}$ & .328 & $\begin{array}{l}.054- \\
1.97\end{array}$ & & .840 & $\begin{array}{l}.437- \\
1.61\end{array}$ & 1.35 & $\begin{array}{l}.593- \\
3.09\end{array}$ & .467 & $\begin{array}{l}.156- \\
1.39\end{array}$ \\
\hline \multicolumn{14}{|c|}{$\begin{array}{l}\text { Lost job due to COVID- } \\
19\end{array}$} \\
\hline No (176) & 1.00 & reference & 1.00 & reference & 1.00 & reference & .147 & 1.00 & reference & 1.00 & reference & 1.00 & reference \\
\hline Yes (18) & 1.01 & $\begin{array}{l}.353- \\
2.92\end{array}$ & 2.51 & $\begin{array}{l}.566- \\
11.1\end{array}$ & .338 & $\begin{array}{l}.035- \\
3.26\end{array}$ & & 1.22 & $\begin{array}{l}.412- \\
3.63\end{array}$ & .697 & $\begin{array}{l}.165- \\
2.94\end{array}$ & 4.56 & $\begin{array}{l}.445- \\
46.7\end{array}$ \\
\hline \multicolumn{14}{|c|}{$\begin{array}{l}\text { Change in healthcare } \\
\text { services }\end{array}$} \\
\hline No (139) & 1.00 & reference & 1.00 & reference & 1.00 & reference & .281 & 1.00 & reference & 1.00 & reference & 1.00 & reference \\
\hline Yes (55) & .616 & $\begin{array}{l}.300- \\
1.26\end{array}$ & .874 & $\begin{array}{l}.376- \\
2.03\end{array}$ & .348 & $\begin{array}{l}.080- \\
1.51\end{array}$ & & .712 & $\begin{array}{l}.346- \\
1.46\end{array}$ & .383 & $\begin{array}{l}.137- \\
1.06\end{array}$ & 1.60 & $\begin{array}{l}.491- \\
5.24\end{array}$ \\
\hline
\end{tabular}

Page $12 / 18$ 


\begin{tabular}{|c|c|c|c|c|c|c|c|c|c|c|c|c|c|}
\hline & \multicolumn{2}{|c|}{$\begin{array}{l}\text { All women } \\
(\mathrm{N}=194)\end{array}$} & \multicolumn{2}{|c|}{$\begin{array}{l}\text { Low income } \\
(\mathrm{N}=45)\end{array}$} & \multicolumn{2}{|c|}{$\begin{array}{l}\text { Average/high } \\
\text { income } \\
(\mathrm{N}=149)\end{array}$} & \multirow[t]{2}{*}{ int. } & \multicolumn{2}{|c|}{$\begin{array}{l}\text { All women } \\
(\mathrm{N}=194)\end{array}$} & \multicolumn{2}{|c|}{$\begin{array}{l}\text { Racial/ethnic } \\
\text { minority } \\
(\mathrm{N}=65)\end{array}$} & \multicolumn{2}{|c|}{$\begin{array}{l}\text { NHW } \\
(\mathrm{N}=129)\end{array}$} \\
\hline & $\mathrm{aOR}$ & $95 \% \mathrm{Cl}$ & aOR & $95 \% \mathrm{Cl}$ & aOR & $95 \% \mathrm{Cl}$ & & aOR & $95 \% \mathrm{Cl}$ & aOR & $95 \% \mathrm{Cl}$ & aOR & $95 \% \mathrm{Cl}$ \\
\hline \multicolumn{14}{|c|}{$\begin{array}{l}\text { Stigma or } \\
\text { discrimination }\end{array}$} \\
\hline No (176) & 1.00 & reference & 1.00 & reference & 1.00 & reference & .799 & 1.00 & reference & 1.00 & reference & 1.00 & reference \\
\hline Yes (18) & 4.65 & $\begin{array}{l}1.45- \\
14.8\end{array}$ & 5.61 & $\begin{array}{l}1.28- \\
24.5\end{array}$ & 3.98 & $\begin{array}{l}.468- \\
33.9\end{array}$ & & 4.22 & $\begin{array}{l}1.35- \\
13.2\end{array}$ & 5.95 & $\begin{array}{l}1.55- \\
22.8\end{array}$ & 2.80 & $\begin{array}{l}.284- \\
27.7\end{array}$ \\
\hline \multicolumn{14}{|c|}{ Personal financial loss } \\
\hline No (133) & 1.00 & reference & 1.00 & reference & 1.00 & reference & .121 & 1.00 & reference & 1.00 & reference & 1.00 & reference \\
\hline Yes (61) & .868 & $\begin{array}{l}.441- \\
1.70\end{array}$ & 1.41 & $\begin{array}{l}.630- \\
3.18\end{array}$ & .392 & $\begin{array}{l}.095- \\
1.61\end{array}$ & & .942 & $\begin{array}{l}.472- \\
1.87\end{array}$ & 1.13 & $\begin{array}{l}.479- \\
2.69\end{array}$ & .846 & $\begin{array}{l}.259- \\
2.76\end{array}$ \\
\hline \multicolumn{14}{|c|}{ Frustration/Boredom } \\
\hline No (100) & 1.00 & reference & 1.00 & reference & 1.00 & reference & .122 & 1.00 & reference & 1.00 & reference & 1.00 & reference \\
\hline Yes (94) & 1.48 & $\begin{array}{l}.775- \\
2.85\end{array}$ & 1.99 & $\begin{array}{l}.958- \\
4.13\end{array}$ & .558 & $\begin{array}{l}.132- \\
2.35\end{array}$ & & 1.60 & $\begin{array}{l}.846- \\
3.04\end{array}$ & 1.50 & $\begin{array}{l}.669- \\
3.39\end{array}$ & 1.65 & $\begin{array}{l}.561- \\
4.87\end{array}$ \\
\hline \multicolumn{14}{|c|}{$\begin{array}{l}\text { Not having basic } \\
\text { supplies }\end{array}$} \\
\hline No (175) & 1.00 & reference & 1.00 & reference & 1.00 & reference & .897 & 1.00 & reference & 1.00 & reference & 1.00 & reference \\
\hline Yes (19) & 2.34 & $\begin{array}{l}.807- \\
6.78\end{array}$ & 2.62 & $\begin{array}{l}.670- \\
10.2\end{array}$ & 3.05 & $\begin{array}{l}.493- \\
18.9\end{array}$ & & 2.61 & $\begin{array}{l}.932- \\
7.32\end{array}$ & 2.17 & $\begin{array}{l}.586- \\
8.07\end{array}$ & 5.12 & $\begin{array}{l}.546- \\
48.0\end{array}$ \\
\hline \multicolumn{14}{|c|}{ More anxiety } \\
\hline No (89) & 1.00 & reference & 1.00 & reference & 1.00 & reference & .371 & 1.00 & reference & 1.00 & reference & 1.00 & reference \\
\hline Yes (105) & 1.73 & $\begin{array}{l}.895- \\
3.36\end{array}$ & 2.15 & $\begin{array}{l}1.02- \\
4.56\end{array}$ & 1.05 & $\begin{array}{l}.258- \\
4.35\end{array}$ & & 1.85 & $\begin{array}{l}.963- \\
3.58\end{array}$ & 1.43 & $\begin{array}{l}.626- \\
3.27\end{array}$ & 2.99 & $\begin{array}{l}.997- \\
8.97\end{array}$ \\
\hline \multicolumn{14}{|c|}{ Sleep issues } \\
\hline No (89) & 1.00 & reference & 1.00 & reference & 1.00 & reference & .165 & 1.00 & reference & 1.00 & reference & 1.00 & reference \\
\hline Yes (105) & 2.07 & $\begin{array}{l}1.05- \\
4.06\end{array}$ & 2.95 & $\begin{array}{l}1.36- \\
6.36\end{array}$ & .924 & $\begin{array}{l}.208- \\
4.09\end{array}$ & & 2.10 & $\begin{array}{l}1.07- \\
4.09\end{array}$ & 1.32 & $\begin{array}{l}.571- \\
3.07\end{array}$ & 7.39 & $\begin{array}{l}2.21- \\
24.6\end{array}$ \\
\hline \multicolumn{14}{|c|}{$\begin{array}{l}\text { Increased substance } \\
\text { use }\end{array}$} \\
\hline No (103) & 1.00 & reference & 1.00 & reference & 1.00 & reference & .114 & 1.00 & reference & 1.00 & reference & 1.00 & reference \\
\hline Yes (91) & 2.13 & $\begin{array}{l}.863- \\
5.29\end{array}$ & 3.28 & $\begin{array}{l}1.12- \\
9.61\end{array}$ & .395 & $\begin{array}{l}.035- \\
4.39\end{array}$ & & 2.09 & $\begin{array}{l}.862- \\
5.07\end{array}$ & 1.13 & $\begin{array}{l}.341- \\
3.75\end{array}$ & 9.44 & $\begin{array}{l}1.09- \\
82.5\end{array}$ \\
\hline \multicolumn{14}{|c|}{$\begin{array}{l}\text { Change in sexual } \\
\text { activity }\end{array}$} \\
\hline No (168) & 1.00 & reference & 1.00 & reference & 1.00 & reference & .420 & 1.00 & reference & 1.00 & reference & 1.00 & reference \\
\hline Yes (26) & 1.85 & $\begin{array}{l}.807- \\
4.28\end{array}$ & 1.50 & $\begin{array}{l}.599- \\
3.79\end{array}$ & 3.79 & $\begin{array}{l}.486- \\
29.5\end{array}$ & & 1.78 & $\begin{array}{l}.785- \\
4.06\end{array}$ & 1.51 & $\begin{array}{l}.498- \\
4.59\end{array}$ & 2.19 & $\begin{array}{l}.573- \\
8.41\end{array}$ \\
\hline \multicolumn{14}{|c|}{ Loneliness } \\
\hline No (161) & 1.00 & reference & 1.00 & reference & 1.00 & reference & .325 & 1.00 & reference & 1.00 & reference & 1.00 & reference \\
\hline Yes (33) & 1.85 & $\begin{array}{l}.976- \\
3.53\end{array}$ & 2.31 & $\begin{array}{l}1.10- \\
4.83\end{array}$ & 1.05 & $\begin{array}{l}.264- \\
4.22\end{array}$ & & 2.07 & $\begin{array}{l}1.09- \\
3.94\end{array}$ & 1.67 & $\begin{array}{l}.736- \\
3.79\end{array}$ & 2.57 & $\begin{array}{l}.875- \\
7.59\end{array}$ \\
\hline \multicolumn{14}{|c|}{$\begin{array}{l}\text { Confusion about } \\
\text { COVID-19 }\end{array}$} \\
\hline No (111) & 1.00 & reference & 1.00 & reference & 1.00 & reference & .894 & 1.00 & reference & 1.00 & reference & 1.00 & reference \\
\hline Yes (83) & 1.05 & $\begin{array}{l}.333- \\
3.35\end{array}$ & 1.04 & $\begin{array}{l}.284- \\
3.83\end{array}$ & 1.28 & $\begin{array}{l}.083- \\
19.6\end{array}$ & & 1.01 & $\begin{array}{l}.320- \\
3.19\end{array}$ & 1.55 & $\begin{array}{l}.362- \\
6.66\end{array}$ & .600 & $\begin{array}{l}.088- \\
4.09\end{array}$ \\
\hline \multicolumn{14}{|c|}{$\begin{array}{l}\text { Giving to greater good } \\
\text { by following } \\
\text { mandates }\end{array}$} \\
\hline No (179) & 1.00 & reference & 1.00 & reference & 1.00 & reference & .383 & 1.00 & reference & 1.00 & reference & 1.00 & reference \\
\hline
\end{tabular}




\begin{tabular}{|c|c|c|c|c|c|c|c|c|c|c|c|c|c|}
\hline & \multicolumn{2}{|c|}{$\begin{array}{l}\text { All women } \\
(\mathrm{N}=194)\end{array}$} & \multicolumn{2}{|c|}{$\begin{array}{l}\text { Low income } \\
(\mathrm{N}=45)\end{array}$} & \multicolumn{2}{|c|}{$\begin{array}{l}\text { Average/high } \\
\text { income } \\
(\mathrm{N}=149)\end{array}$} & \multirow[t]{2}{*}{ int. } & \multicolumn{2}{|c|}{$\begin{array}{l}\text { All women } \\
(N=194)\end{array}$} & \multicolumn{2}{|c|}{$\begin{array}{l}\text { Racial/ethnic } \\
\text { minority } \\
(\mathrm{N}=65)\end{array}$} & \multicolumn{2}{|c|}{$\begin{array}{l}\text { NHW } \\
(\mathrm{N}=129)\end{array}$} \\
\hline & aOR & $95 \% \mathrm{Cl}$ & aOR & $95 \% \mathrm{Cl}$ & aOR & $95 \% \mathrm{Cl}$ & & aOR & $95 \% \mathrm{Cl}$ & aOR & $95 \% \mathrm{Cl}$ & aOR & $95 \% \mathrm{Cl}$ \\
\hline Yes (15) & .518 & $\begin{array}{l}.258- \\
1.04\end{array}$ & .661 & $\begin{array}{l}.297- \\
1.46\end{array}$ & .308 & $\begin{array}{l}.066- \\
1.42\end{array}$ & & .462 & $\begin{array}{l}.233 \\
-.915\end{array}$ & .572 & $\begin{array}{l}.249- \\
1.31\end{array}$ & .519 & $\begin{array}{l}.137- \\
1.96\end{array}$ \\
\hline
\end{tabular}

Getting emotional

support from loved

ones

\begin{tabular}{|c|c|c|c|c|c|c|c|c|c|c|c|c|c|}
\hline No (128) & 1.00 & reference & 1.00 & reference & 1.00 & reference & .913 & 1.00 & reference & 1.00 & reference & 1.00 & reference \\
\hline Yes (66) & .773 & $\begin{array}{l}.380- \\
1.57\end{array}$ & .814 & $\begin{array}{l}.360- \\
1.83\end{array}$ & .741 & $\begin{array}{l}.168- \\
3.26\end{array}$ & & .805 & $\begin{array}{l}.399- \\
1.62\end{array}$ & .764 & $\begin{array}{l}.314- \\
1.85\end{array}$ & .862 & $\begin{array}{l}.257- \\
2.88\end{array}$ \\
\hline
\end{tabular}

Getting financial

support from loved

ones

\begin{tabular}{|c|c|c|c|c|c|c|c|c|c|c|c|c|c|}
\hline No (140) & 1.00 & reference & 1.00 & reference & 1.00 & reference & .101 & 1.00 & reference & 1.00 & reference & 1.00 & reference \\
\hline Yes (54) & .816 & $\begin{array}{l}.276- \\
2.41\end{array}$ & 4.01 & $\begin{array}{l}.617- \\
26.0\end{array}$ & .533 & $\begin{array}{l}.166- \\
2.43\end{array}$ & & 1.12 & $\begin{array}{l}.372- \\
3.41\end{array}$ & 1.64 & $\begin{array}{l}.473- \\
5.74\end{array}$ & .467 & $\begin{array}{l}.060- \\
3.58\end{array}$ \\
\hline
\end{tabular}

Missing values: change in life due to COVID-19 (15), age (3), and cancer status (3).

Bold font indicates statistically significant with corresponding $p<.05$.

p-interaction terms are between income status and predictor(s).

Covariates/stratifications: age (continuous), number of comorbid conditions (continuous), years since genetic testing (continuous), education (some college less, college graduate or above), marital status (married/living as married, other), cancer status (no cancer history, cancer history), income status (average/hi income, low income), and race/ethnicity (non-Hispanic white [NHW], Hispanic or racial minority).

\section{Discussion}

Among BRCA1/2-positive women residing in the US, the current study analyzed relationships between experiencing COVID-19-related instances and odds of reporting anxiety and depression overall and stratified by sociodemographic factors. Demographically, most of the sample was younger than age 50 , consistent with past literature suggesting that women are being genetically tested at younger ages ${ }^{38,39}$. Most women were NHW and educated, but there was some diversity where as much as $40.8 \%$ reported a physical disability and $61.6 \%$ a chronic condition. The current study is novel in its relation to COVID-19, however research remains limited regarding the pandemic and its impact on at-risk cancer populations such as those with $B R C A 1 / 2$ mutations.

Commonalities existed with several COVID-19-related experiences predicting increases in anxiety and depression symptomologies. It appears reporting stigma or discrimination, sleep issues, or increased substance use during the pandemic resulted in significantly increased chances of having more anxiety and depression symptoms than women who did not report these instances. Although it is well-known that BRCA1/2-positive women report on average, higher levels of anxiety and depression than the general population, these increases have not been directly connected to the COVID-19 pandemic, but within past literature have focused on the stress of ongoing surveillance and prophylactic risk-reducing surgeries ${ }^{21,40,41}$ and cancer patients generally ${ }^{42}$. Not surprisingly, there were differences in income status, where women with average/high income were less likely to report depressive symptoms if they quarantined due to COVID-19. As we know, individuals who have both the resources and time to seek mental healthcare are more likely to utilize such care ${ }^{43,44}$, but does not account for COVID-19-related barriers. Interestingly, for those caring for someone at home during the pandemic, there were differences by race/ethnicity, where $\mathrm{NHW}$ were less likely to experience depression, but minority women were almost three times more likely. Past literature has found that among caregivers, depression and anxiety were higher in Black or African Americans than $\mathrm{NHW}$ women ${ }^{45}$, but other literature has reported that mental health symptoms increased with level of care ${ }^{46}$. Due to the recency of the COVID-19 pandemic in conjunction with its effect on both cancer patients, survivors, and those at increased risk for cancer like the women in this study, this topic remains relatively new and suggests the importance of researching this further.

To our knowledge, no studies have been conducted focusing on BRCA1/2-positive women's mental health and their relation to the COVID-19 pandemic. In current literature, healthcare utilization in relation to genetic testing ${ }^{47}$ and cancer-related diagnostic delays ${ }^{48}$ has been introduced in recent years, but not many have highlighted how the pandemic has impacted cancer patients or survivors' mental health. In one such study, Wang and colleagues ${ }^{30}$ published that among 6213 cancer patients, $23.4 \%$ experienced depression and $17.7 \%$ had anxiety. In relation to COVID-19, individuals showing a history of mental health adversities, alcohol consumption, and continuous cancer worry were predominant factors for mental health symptomology among this population ${ }^{30}$. The recent pandemic's impact on the mental health of the general population has been published more often, noting that both the direct and indirect psychological impact of COVID-19 on the general public and vulnerable groups (e.g., elderly, people with pre-existing mental health issues, etc.) ${ }^{49}$ should be studied in more detail. Similarly, symptoms of mental health during COVID-19 have been exacerbated by lower quality of life and focusing on the negative aspects of the pandemic ${ }^{50}$. The recency of the COVID-19 pandemic in the US has focused research on the general population and its mental health, while very little, to our knowledge, has been implemented among cancer patients or survivors, and none regarding BRCA1/2-positive women.

It is apparent that the COVID-19 pandemic had variable effects on certain groups such as BRCA1/2-positive racial/ethnic minority women and those with low income. While research is continuing to emerge in response to the COVID-19 pandemic in relation to cancer and cancer risk, future studies should focus on 
stratifying by groups who are at higher risk for cancer and those who have survived it. Larger, more inclusive nationwide studies may provide the framework necessary to distinctly analyze subgroups such as these so resources following this pandemic may be of benefit to all in the US. Longitudinal studies could be implemented to discover the impact of COVID-19 on the cancer care continuum, from screening to survivorship. Resources should be made available to individuals experiencing compounded disparities, like those mentioned in the current study, to help alleviate the adverse mental health symptoms that may arise due to COVID-19, surveillance, and surgery. The National Cancer Institute (NCI) ${ }^{51}$ and American Cancer Society (ACS) ${ }^{28,52}$, and even several large hospital systems such as Johns Hopkins Medicine in collaboration with the National Comprehensive Cancer Network (NCCN) ${ }^{53}$ have published websites to assist cancer patients and survivors navigate the COVID-19 pandemic. Clinically, mental health screening at routine healthcare appointments may be beneficial to this population in combination with available mental health resources and recommendations. However, because this is a new realm of research, additional research is needed to accurately describe the relationship between COVID-19, anxiety, and depression among at-risk cancer groups such as women with BRCA1/2 mutations.

\section{Study strengths.}

The current study has several strengths. Our study attempted to recruit from a combination of hard-to-reach populations and those with rare cancer hereditary genetic mutations not easily recruited in-person. The online nature of this study acted as a pilot to test if these populations could be recruited successfully and from areas across the US. We were able to recruit a female sample from diverse backgrounds, allowing for limited generalizability to subpopulations such as racial/ethnic minorities, those with low income, and those with cancer. Future studies can use these approaches to recruit other hard-to-reach populations for rare or stigmatized health conditions.

\section{Limitations.}

The current study's findings should be interpreted with consideration of its limitations. Overall, while the current study provided a moderately large sample, the data is cross-sectional and self-reported, which may introduce misclassification or recall bias. Stratified results should be interpreted with caution due to limited sample sizes among the subgroups of interest. Our findings should be replicated in a larger study with a similar study population to confirm similarities. It is also possible that by using predictors that were originally dichotomous may limit the implication of detailed information, as future studies may ask about the severity of COVID-19 experiences in addition to incidence. These participants were recruited from online support groups, which may introduce bias by being more open and willing to share experiences than others not in support groups ${ }^{54}$. Therefore, generalization of these findings is limited to the populations analyzed in the current sample.

\section{Conclusion}

The current study provides a unique view in beginning to understand the impact of the COVID-19 pandemic on anxiety and depression among women with BRCA1/2 mutations. This perspective allowed the identification of several COVID-19-related experiences in relation to mental health outcomes, stratified by income status and race/ethnicity, showing that there are distinct disparities among both groups. Future research can target the development of anxiety and depressive symptom relief during and after the COVID-19 pandemic utilizing prospective longitudinal study designs, while interventions can focus on recurrent training for medical professionals working with this population. Clinically, medical professionals should offer referrals to mental health counseling for all patients, not only those who are visibly struggling during this pandemic. With genetic testing becoming more widely available, especially with the utilization of telemedicine, it is possible that women may require ongoing mental healthcare that are not currently widely available for those of low income and racial/ethnic minority groups to reduce the inequities among those with $B R C A 1 / 2$ mutations.

\section{Declarations}

\section{Funding:}

The current study was funded by the Johns Hopkins Ho-Ching Yang Memorial Faculty Award. KD received research support from the National Cancer Institute (T32CA009314) Cancer Epidemiology, Prevention, and Control training program.

\section{Availability of data and material:}

The datasets generated during and/or analyzed the current study are available at the Principal Investigator (PI)'s discretion upon reasonable request.

\section{Code availability:}

Syntax coding is available upon reasonable request from the corresponding author.

\section{Author contributions}

Kate E Dibble conceptualized and designed the study, was in charge of data acquisition, data analysis, and interpretation. Kate E Dibble also wrote the main manuscript text and revised the article, as well as approving the final version. Avonne E Connor assisted with study conceptualization and design, data 
interpretation, as well as drafting and finalizing the manuscript.

\section{Ethics approval:}

This study was performed in line with the principles of the Declaration of Helsinki. Approval was granted by the Johns Hopkins Bloomberg School of Public Health Institutional Review Board.

\section{Consent to participate:}

Informed consent was obtained from all individual participants included in the study.

\section{Consent for publication:}

Not applicable

\section{Competing interests:}

Dr. Kate Dibble has no conflicts of interest to disclose. Dr. Avonne Connor has no conflicts of interest to disclose.

\section{References}

1. American Cancer Society (ACS). Family cancer syndromes. https://www.cancer.org/cancer/cancer-causes/genetics/family-cancer-syndromes.html (2020).

2. Godet, I., \& Gilkes, D. M. BRCA1 and BRCA2 mutations and treatment strategies for breast cancer. Integrative Cancer Science and Technologies. 4, 10 (2017).

3. Mersch, J.et al. Cancers associated with BRCA1 and BRCA2 mutations other than breast and ovarian. Cancer. 121, 269-275, https://doi.org/10.1002/cncr.29357 (2015).

4. Kuchenbaecker, K. B.et al. Risks of breast, ovarian, and contralateral breast cancer for BRCA1 and BRCA2 mutation carriers. JAMA. 317, https://doi.org/10.1001/jama.2017.7112 (2017).

5. Haque, R.et al. Survival outcomes in BRCA1 or BRCA2 mutation carriers and the influence of triple-negative breast cancer subtype. The Permanente Journal. 22, 170-197, https://dx.doi.org/10.7812\%2FTPP\%2F17-197 (2018).

6. Song, Y.et al. Patterns of recurrence and metasasis in BRCA1/BRCA2-associated breast cancers. Cancer. 126, 271-280, https://doi.org/10.1002/cncr.32540 (2020).

7. Mau, C. \& Untch, M. Prophylactic surgery: For whom, when and how. Breast Care. 12, 379-384, https://doi.org/10.1159/000485830 (2017).

8. Ludwig, K. K., Neuner, J., Butler, A., Geurts, J. L. \& Kong, A. L. Risk reduction and survival benefit of prophylactic surgery in BRCA mutation carriers, a systematic review. The American Journal of Surgery. 212, 660-669, https://doi.org/10.1016/j.amjsurg.2016.06.010 (2016).

9. van Zelst, J. C. M.et al. Surveillance of women with the BRCA1 or BRCA2 mutation by using biannual automated breast US, MR imaging, and mammography. Radiology. 285, 376-388, https://doi.org/10.1148/radiol.2017161218 (2017).

10. Nelson, H. D., Pappas, M., Cantor, A., Haney, E. \& Holmes, R. Risk assessment, genetic counseling, and genetic testing for BRCA-related cancer in women: Updated evidence report and systematic review for the US Preventive Services Task Force. JAMA. 322, 666-685, https://doi.org/10.1001/jama.2019.8430 (2019).

11. Segerer, R.et al. Factors impacting on decision-making towards prophylactic surgeries in BRCA mutation carriers and women with familial predisposition. Breast Care. 15, 253-259, https://doi.org/10.1159/000503370 (2020).

12. Gopie, J. P., Vasen, H. F. A. \& Tibben, A. Surveillance for hereditary cancer: Does the benefit outweigh the psychological burden? A systematic review. Critical Reviews in Oncology/Hematology. 83, 329-340, https://doi.org/10.1016/j.critrevonc.2012.01.004 (2012).

13. Borreani, C.et al. The psychological impact of breast and ovarian cancer preventive options in BRCA1 and BRCA2 mutation carriers. Clinical Genetics. 85 , 7-15, https://doi.org/10.1111/cge.12298 (2013).

14. Harmsen, M. G., Hermens, R. P. M. G., Prins, J. B., Hoogerbrugge, N., \& de Hullu, J. A. How medical choices influence quality of life of women carrying a BRCA mutation. Critical Reviews in Oncology/Hematology. 96, 555-568 (2015).

15. Razdan, S. N., Patel, V., Jewell, S., \& McCarthy, C. M. . Quality of life among patients after bilateral prophylactic mastectomy: A systematic review of patient-reported outcomes. Quality of Life Research. 25, 1409-1421 (2016).

16. Portnoy, D. B., Loud, J. T., Han, P. K. J., Mai, P. L. \& Greene, M. H. Effects of false-positive cancer screenings and cancer worry on risk-reducing surgery among BRCA1/2 carriers. Health Psychology. 34, 709-717, https://psycnet.apa.org/doi/10.1037/hea0000156 (2015).

17. Rush, S. K.et al. Cancer worry and decision making about risk reduction in women with BRCA1 and BRCA2 mutations. Gynecologic Oncology. 156, E29, https://doi.org/10.1016/j.ygyno.2019.11.090 (2020). 
18. Hoberg-Vetti, H.et al. BRCA1/2 testing in newly diagnosed breast and ovarian cancer patients without prior genetic counseling: The DNA-BONus study. European Journal of Human Genetics. 24, 881-888, https://doi.org/10.1038/ejhg.2015.196 (2016).

19. Mella, S., Muzzatti, B., Dolcetti, R. \& Annunziata, M. A. Emotional impact on the results of BRCA1 and BRCA2 genetic test: An observational retrospective study. Hereditary Cancer in Clinical Practice. 15, 16, https://doi.org/10.1186/s13053-017-0077-6 (2017).

20. den Heijer, M.et al. Long-term psychological distress in women at risk for hereditary breast cancer adhering to regular surveillance: A risk profile. PsychoOncology. 22, 598-604, https://doi.org/10.1002/pon.3039 (2012).

21. Ringwald, J.et al. Psychological Distress, Anxiety, and Depression of Cancer-Affected BRCA1/2 Mutation Carriers: a Systematic Review. Journal of Genetic Counseling 25, 880-891, https://doi.org/10.1007/s10897-016-9949-6 (2016).

22. Bakouny, Z.et al. Cancer screening tests and cancer diagnoses during the COVID-19 pandemic. JAMA Oncology. 7, 458-460, http://jamanetwork.com/article.aspx?doi=10.1001/jamaoncol.2020.7600 (2021).

23. Calvo, R. A., Deterding, S. \& Ryan, R. M. Health surveillance during COVID-19 pandemic. BMJ. 369, m1373, https://doi.org/10.1136/bmj.m1373 (2020).

24. Kaufman, H. W., Chen, Z., Niles, J. \& Fesko, Y. Changes in the number of US patients with newly identified cancer before and during the Coronavirus Disease 2019 (COVID-19) pandemic. JAMA Network Open. 3, e2017267, http://jamanetwork.com/article.aspx? doi=10.1001/jamanetworkopen.2020.17267 (2020).

25. Jee, J.et al. Chemotherapy and COVID-19 outcomes in patients with cancer. Journal of Clinical Oncology. 38, 3538-3546, https://doi.org/10.1200/jco.20.01307 (2020).

26. Robilotti, E. V.et al. Determinants of COVID-19 disease severity in patients with cancer. Nature Medicine. 26, 1218-1223, https://doi.org/10.1038/s41591020-0979-0 (2020).

27. Williamson, E. J.et al. Factors associated with COVID-19-related death using OpenSAFELY. Nature. 584, 430-436, https://doi.org/10.1038/s41586-0202521-4 (2020).

28. American Cancer Society (ACS). Special section: COVID-19 and cancer, https://www.cancer.org/content/dam/cancer-org/research/cancer-facts-andstatistics/annual-cancer-facts-and-figures/2021/special-section-covid19-and-cancer-2021.pdf (2021).

29. Epic Health Research Network. Preventive cancer screenings during COVID-19 pandemic, https://ehrn.org/articles/delays-in-preventive-cancer-screeningsduring-covid-19-pandemic/ (2020).

30. Wang, Y.et al. Epidemiology of mental health problems among patients with cancer during COVID-19 pandemic. Translational Psychiatry. 10, 263, https://doi.org/10.1038/s41398-020-00950-y (2020).

31. Harris, P. A.et al. Research electronic data capture (REDCap) - A metadata-driven methodology and workflow process for providing translational research informatics support. Journal of Biomedical Informatics. 42, 377-381, https://doi.org/10.1016/j.jbi.2008.08.010 (2009).

32. Harris, P. A.et al. The REDCap consortium: Building an international community of software platform partners. Journal of Biomedical Informatics. 95 , 103208, https://doi.org/10.1016/j.jbi.2019.103208 (2019).

33. Harkness, A. The pandemic stress index, https://elcentro.sonhs.miami.edu/research/measures-library/psi/psi-english/index.html (2020).

34. Spitzer, R. L., Kroenke, K. \& Williams, J. B. W. A brief measure for assessing generalized anxiety disorder. Archives of Internal Medicine. 166, 1092-1097, https://doi.org/10.1001/archinte.166.10.1092 (2006).

35. Lowe, B.et al. Validation and standardization of the Generalized Anxiety Disorder screener (GAD-7) in the general population. Medical Care. 46, 266-274, https://doi.org/10.1097/mlr.0b013e318160d093 (2008).

36. Kroenke, K., Spitzer, R. L. \& Williams, J. B. W. The PHQ-9: Validity of a brief depression severity measure. Journal of General Internal Medicine. 16, 606-613, https://dx.doi.org/10.1046\%2Fj.1525-1497.2001.016009606.x (2001).

37. Stata Statistical Software: Release 16 v. 16 (College Station, TX, 2019).

38. Guo, F., Scholl, M., Fuchs, E. L., Berenson, A. B. \& Kuo, Y. F. BRCA testing in unaffected young women in the United States, 2006-2017. Cancer. 126, 337343, https://doi.org/10.1002/cncr.32536 (2019).

39. Brunstrom, K., Murray, A., \& McAllister, M. Experiences of women who underwent predictive BRCA $1 \frac{12}{2}$ mutation testing before the age of 30 . Journal of Genetic Counseling. 25, 90-100 (2016).

40. Spiegel, T. N.et al. Psychological impact of recall on women with BRCA mutations undergoing MRI surveillance. The Breast. 20, 424-430, https://doi.org/10.1016/j.breast.2011.04.004 (2011).

41. Lombardi, L.et al. Psychological aspects, risk and protective factors relating to BRCA genetic testing: A review of the literature. Supportive Care in Cancer. 27, 3647-3656, https://doi.org/10.1007/s00520-019-04918-7 (2019).

42. Niedzwiedz, C. L., Knifton, L., Robb, K. A., Katikireddi, S. V. \& Smith, D. J. Depression and anxiety among people living with and beyond cancer: A growing clinical and research priority. BMC Cancer. 19, 943, https://doi.org/10.1186/s12885-019-6181-4 (2019).

43. Crumb, L., Mingo, T. M. \& Crowe, A. "Get over it and move on": The impact of mental illness stigma in rural, low-income United States populations. Mental Health \& Prevention. 13, 143-148, https://doi.org/10.1016/j.mhp.2019.01.010 (2019).

44. Liu, J., Jiang, N., Fan, A. Z. \& Weissman, R. Alternatives in assessing mental healthcare disparities using the Behavioral Risk Factor Surveillance System. Health Equity. 2, 199-206, https://doi.org/10.1089/heq.2017.0056 (2018).

45. Ejem, D., Bauldry, S., Bakitas, M. \& Drentea, P. Caregiver burden, caregiver recipient depressive symptomology, and social exchange: Does race matter? Journal of Palliative Care. 33, 100-108, https://doi.org/10.1177\%2F0825859718758120 (2018). 
46. Cohen, S. A., Sabik, N. J., Cook, S. K., Azzoli, A. B. \& Mendez-Luck, C. A. Differences within differences: Gender inequalities in caregiving intensity vary by race and ethnicity in informal caregivers. Journal of Cross-Cultural Gerontology. 34, 245-263, https://doi.org/10.1177\%2F0825859718758120 (2019).

47. Minucci, A.et al. BRCA testing delay during the COVID-19 pandemic: How to act? Molecular Biology Reports. 48, 983-987, https://doi.org/10.1007/s11033020-06060-8 (2021).

48. Wang, Q. Q., Berger, N. A. \& Xu, R. Analyses of risk, racial disparity, and outcomes among US patients with cancer and COVID-19 infection. JAMA Oncology. 7, 220-227, https://doi.org/10.1001/jamaoncol.2020.6178 (2021).

49. Tsamakis, K.et al. COVID-19 and its consequences on mental health. Experimental and Therapeutic Medicine. 21, 1-7, https://doi.org/10.3892/etm.2021.9675 (2021).

50. Shamblaw, A. L., Rumas, R. L. \& Best, M. W. Coping during the COVID-19 pandemic: Relations with mental health and quality of life. Canadian Psychology/Psychologie Canadienne. 62, 92-100, https://psycnet.apa.org/doi/10.1037/cap0000263 (2021).

51. National Cancer Institute (NCl). Coronavirus and cancer, https://www.cancer.gov/about-cancer/coronavirus (2021).

52. American Cancer Society (ACS). Coronavirus, COVID-19, and cancer, https://www.cancer.org/about-us/what-we-do/coronavirus-covid-19-and-cancer.html (2021).

53. National Comprehensive Cancer Network (NCCN). COVID-19 resources, https://www.nccn.org/covid-19 (2021).

54. Houlihan, M. C. \& Tariman, J. D. Comparison of outcome measures for traditional and online support groups for breast cancer patients: An integrative literature review. Journal of Advanced Practitioner in Oncology. 8, 348-359 (2017). 